\title{
Functional expression of opioid receptors and other human GPCRs in yeast engineered to produce human sterols
}

\author{
Björn D.M. Bean ${ }^{1,+}$, Colleen J. Mulvihill ${ }^{2,+}$, Riddhiman K. Garge ${ }^{2}$, Daniel R. Boutz ${ }^{2,3}$, Olivier Rousseau ${ }^{1}$, \\ Brendan M. Floyd ${ }^{2}$, William Cheney ${ }^{1}$, Elizabeth C. Gardner ${ }^{2}$, Andrew D. Ellington ${ }^{2}$, Edward M. Marcotte ${ }^{2}$, \\ Jimmy D. Gollihar ${ }^{2,3,4^{*}}$, Malcolm Whiteway ${ }^{1}$, Vincent J.J. Martin ${ }^{1 *}$ \\ ${ }^{1}$ Department of Biology, Centre for Applied Synthetic Biology, Concordia University, Montréal, QC, H4B1R6, Canada \\ 2Department of Molecular Biosciences, Center for Systems and Synthetic Biology, The University of Texas at Austin, Austin, \\ Texas, 78712, USA \\ ${ }^{3}$ DEVCOM Army Research Laboratory-South, Austin, 78712, TX, USA \\ ${ }^{4}$ Center for Molecular and Translational Human Infectious Diseases Research, Department of Pathology and Genomic Medicine, \\ Houston Methodist Research Institute, Houston Methodist Hospital, Houston, TX, USA \\ + Equal contribution \\ * Corresponding authors
}

Keywords: humanized yeast, opioid biosensor, G protein-coupled receptors, sterol pathway engineering. 


\section{ABSTRACT}

The yeast Saccharomyces cerevisiae is a powerful tool for studying G protein-coupled receptors (GPCRs) as they can be functionally coupled to its pheromone response pathway. However, some exogenous GPCRs, including the mu opioid receptor, are non-functional in yeast, which may be due to the presence of the fungal sterol ergosterol instead of the animal sterol cholesterol. We engineered yeast to produce cholesterol and introduced the human mu opioid receptor, creating an opioid biosensor capable of detecting the peptide DAMGO at an $\mathrm{EC}_{50}$ of $62 \mathrm{nM}$ and the opiate morphine at an $\mathrm{EC}_{50}$ of $882 \mathrm{nM}$. Furthermore, introducing $\mathrm{mu}$, delta, and kappa opioid receptors from diverse vertebrates consistently yielded active opioid biosensors that both recapitulated expected agonist binding profiles with $\mathrm{EC}_{50} \mathrm{~S}$ as low as 2.5 $\mathrm{nM}$ and were inhibited by the antagonist naltrexone. Additionally, clinically relevant human mu opioid receptor alleles, or variants with terminal mutations, resulted in biosensors that largely displayed the expected changes in activity. We also tested mu opioid receptor-based biosensors with systematically adjusted biosynthetic intermediates of cholesterol, enabling us to relate sterol profiles with biosensor sensitivity. Finally our cholesterol-producing biosensor background was applied to other human GPCRs, resulting in SSTR5, 5-HTR4, FPR1 and NPY1R signaling with varying degrees of cholesterol dependence. Our steroloptimized platform will be a valuable tool in generating human GPCRbased biosensors, aiding in ongoing receptor deorphanization efforts, and providing a framework for high-throughput screening of receptors and effectors.

\section{ABBREVIATIONS}

au, arbitrary units; aMF, alpha mating factor; DAMGO, [D-Ala ${ }^{2}, \mathrm{~N}-$ $\mathrm{MePhe}^{4}$, Gly-ol]-enkephalin; DOR, delta opioid receptor; ER, endoplasmic reticulum; $\mathrm{G}$ protein, guanine nucleotide-binding protein; GPCR, G protein-coupled receptor; KOR, kappa opioid receptor; MOR, mu opioid receptor; PRP, mating pheromone response pathway; SD, standard deviation; SEM, standard error of the mean; tmh, transmembrane helix

\section{INTRODUCTION}

G-protein coupled receptors (GPCRs) detect diverse extracellular stimuli, modulating signal transduction pathways that allow cells to respond to their environment. These seven transmembrane domain proteins typically function by binding external ligands, which induce conformational changes, propagating a signal across the plasma membrane and triggering internal signaling pathways ${ }^{1}$. Owing to their critical functions and their ubiquity as the largest family of human membrane proteins, one third of current FDA approved therapeutic targets are GPCRs ${ }^{2}$. Yet, while these targets are functionally understood, discovery of new GPCR-interacting therapeutics remains challenging in part due to screening limitations.

Assays of GPCR activity in the yeast $S$. cerevisiae may accelerate the search for therapeutics by allowing simple, cheap, and high throughput screens. Commonly, these assays are based on functionally linking GPCRs to the yeast pheromone response pathway (PRP). Normally in the PRP, a native GPCR binds a mating pheromone, causing a GTP-GDP substitution in the Ga protein Gpal, triggering a mitogen activated protein kinase signaling cascade culminating in upregulation of Ste12-regulated genes ${ }^{3,4}$. This pathway can be commandeered to make a biosensor by replacing the native GPCR, creating a chimeric Gpal to maintain the GPCR-G protein interaction, and placing a reporter under the control of a Ste12-regulated promoter ${ }^{5}$. Such yeast-based biosensor designs, initially applied to the $\beta 2$-adrenergic receptor ${ }^{6}$, have now been used for over 50 receptors ${ }^{7}$. Yet, in many cases GPCRs cannot be functionally expressed in yeast $t^{8-10}$. This may be due to poor expression or folding ${ }^{11-13}$, defects in trafficking to the plasma membrane ${ }^{10}$ or differences in the chemical environment ${ }^{14-16}$.

In particular, the function of heterologously-expressed human GPCRs may be disrupted by the membrane lipid composition in yeast, as the dominant sterol is ergosterol, as opposed to cholesterol9. This would be consistent with past work documenting the importance of cholesterol-GPCR interactions ${ }^{17-19}$ and the frequent presence of cholesterol molecules as elements of established GPCR structures ${ }^{20}$. Thus, modifying the sterol profile of yeast may increase the proportion of human GPCRs that can be functionally expressed. Previously, deletion of the ergosterol biosynthetic genes ERG5/6, and introduction of zebrafish enzymes DHCR7/24 resulted in yeast producing cholesterol up to $96 \%$ of total sterol content ${ }^{21,22}$. While this modification disrupted the function of the endogenous GPCR Ste2 ${ }^{21}$, its effect on heterologous GPCRs has not been tested.

It would be valuable to apply yeast-based rapid screening approaches to human opioid receptors. The main opioid receptor types, mu, delta and kappa, are all GPCRs implicated in nociception and analgesia ${ }^{23}$. Drugs targeting these receptors, and the human mu opioid receptor (HsMOR) in particular, are essential front-line pain treatment medicines, but have also enabled misuse and dependence ${ }^{24}$. Expansion of available drugs that target these receptors but lack the side-effects of prototypical opioids could help resolve these issues. Though an HsMORbased biosensor would provide a powerful tool for identifying new drug candidates, past efforts to construct this tool have failed in part due to the sterol composition of yeast membranes leading to low HsMOR activity 9 .

Here we describe a new biosensor background based on signaling through the PRP in a yeast strain engineered to produce cholesterol. This background dramatically improves HsMOR activity relative to an ergosterol-rich strain, enabling the characterization of structural and clinically-relevant HsMOR variants. We probed the agonist sensitivities of opioid biosensors based on 15 different receptors and found that opioid receptor activity and agonist specificities are well conserved in yeast. Screening a library of HsMOR-based biosensors with different sterol profiles allowed us to uncover how cholesterol intermediates affect signaling and establish that the cholesterol producing background was highly effective. Lastly, we applied the cholesterol-producing background as a platform to study other human GPCRs.

\section{RESULTS}

\section{Construction of an opioid biosensor in a cholesterol-producing yeast}

Previous work found that yeast-expressed human mu opioid receptor (HsMOR) was only active in lysates when ergosterol was removed and cholesterol added ${ }^{9}$. Therefore, we investigated whether HsMOR may be active in yeast cells engineered to produce cholesterol instead of ergosterol, and if active, whether linking HsMOR to the PRP would create an opioid biosensor.

We made a biosensor chassis based on previous studies linking GPCRs to the $\mathrm{PRP}^{5,25}$ (Figure 1A). The pheromone receptor, Ste2, was 
a Pheromone Response Pathway a pheromone
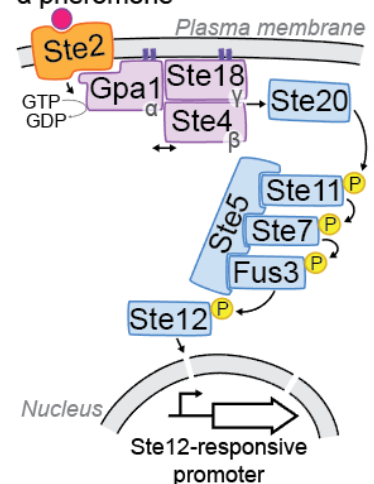

C 8

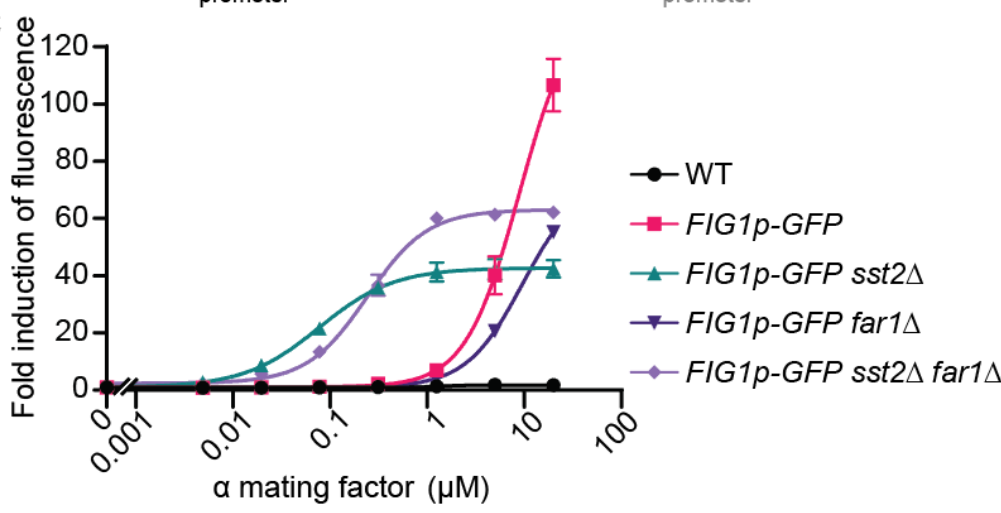

$\mathbf{e}$

wild type

$\operatorname{erg} 5 \Delta:: D r . D H C R 7$

$\operatorname{erg} 6 \triangle:: D r . D H C R 24$

sterol mutant

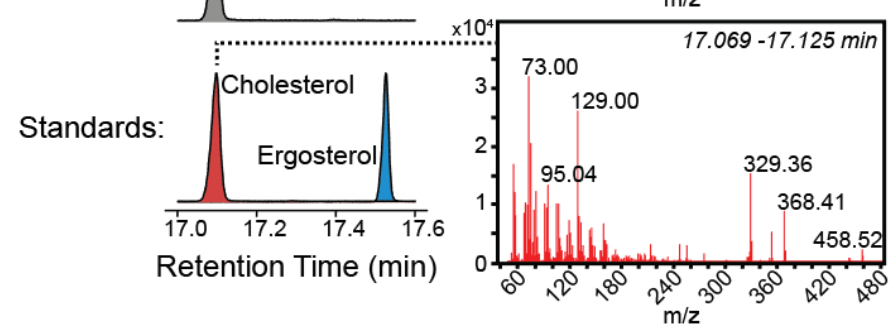

b

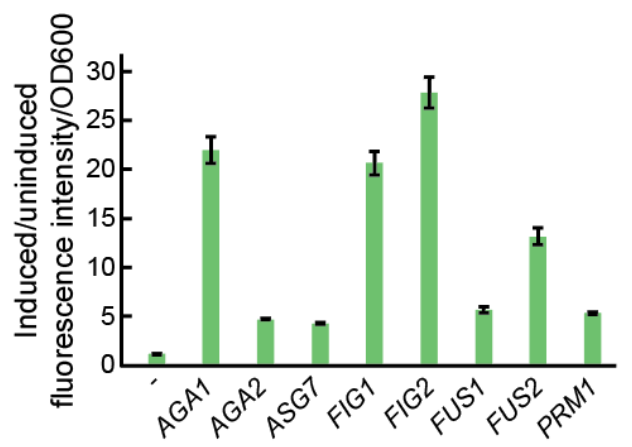

Promoter driving GFP expression
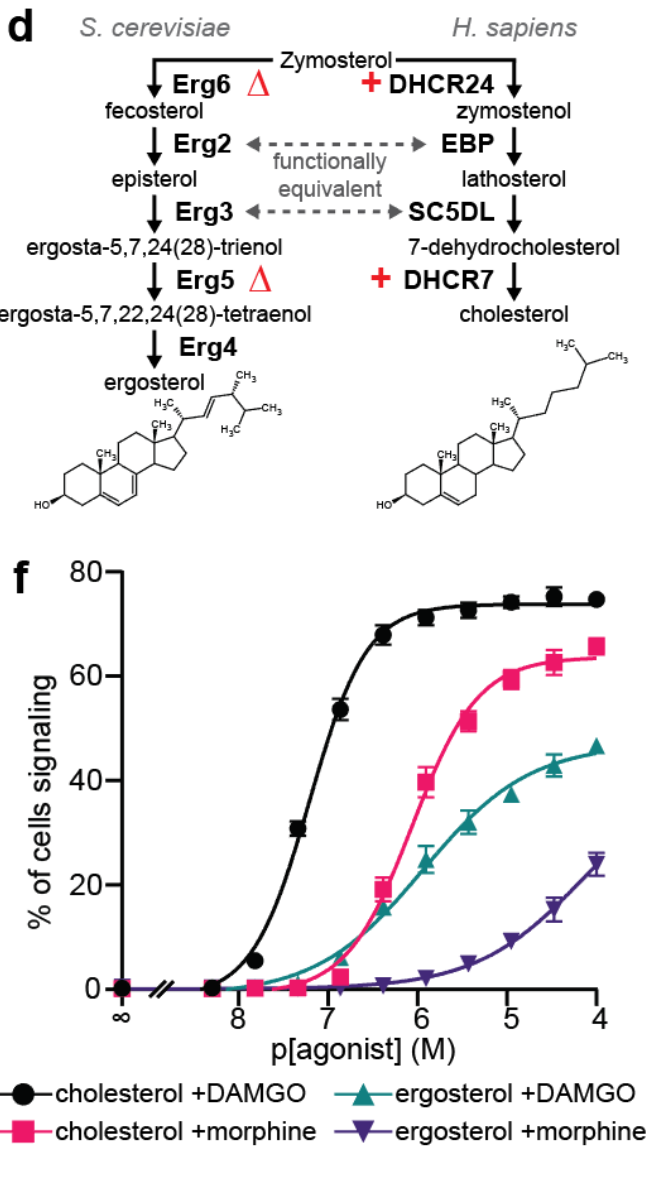

Figure 1. Development of a yeast-based opioid biosensor. (a) Strategy to adapt the yeast pheromone response pathway (PRP, left) into a biosensor pathway (right). An exogenous GPCR is introduced and linked to the pathway by a Gpa1 chimera. Deletion of SST2 and FAR1 respectively potentiates signaling and blocks signaling-induced cell cycle arrest. Sterols are converted to cholesterol and a promoter controlled by the PRP-responsive Ste12 transcription factor drives a GFP output. (b) Biosensor reporter promoters tested using alpha mating factor-induced GFP expression from yeast Ste12-responsive promoters after $3 \mathrm{~h}$ treatment. $\mathrm{n}=3$. (c) PRP sensitivity and activity resulting from SST2 and/or FAR1 deletions. Strains were treated with alpha mating factor for $6 \mathrm{~h}$, mean fluorescence was measured by flow cytometry. $n=3 ; 10000$ cells/strain/replicate. (d) Ergosterol and cholesterol biosynthetic pathways from zymosterol. (e) GC-MS analysis of sterol extracts showing successful synthesis of cholesterol in an erg5/6 DHCR7/24 background. Chromatograms indicating retention times of derivatized sterols from a wild-type strain (top), the engineered strain (middle), and standards (bottom). MS spectra extracted from engineered strain (top) and cholesterol standard (bottom). (f) DAMGO and morphine dose-response curves for ergosterol and cholesterol-producing HsMOR-based biosensors. Measured by flow cytometry after $8 \mathrm{~h}$ agonist treatment. $\mathrm{n}=3 ; 10000$ cells/strain/replicate. Error bars indicate SEM.

removed to avoid interference and the final five residues of the $\mathrm{Ga}$ protein, Gpal, were swapped with the endogenous HsMOR-interacting protein $\mathrm{G}_{\mathrm{ia} 3}\left(\mathrm{~K}_{468} \mathrm{IGII}>\mathrm{ECGLY}\right)$ to generate a chimera previously shown to link exogenous GPCRs to the PRP5 . We chose green fluorescent protein (GFP) expression as an output and selected the promoter 3 controlling expression by using alpha mating factor to screen the ability of eight highly PRP-regulated promoters to express $\mathrm{GFP}^{26,27}$ (Figure 1B). While pFUS1 is often used ${ }^{5,6,28}$, we found that $p A G A 1, p F I G 1$ and $p F I G 2$ all yield roughly four times the response, leading us to select $p F I G 1:: G F P$ as the reporter. 
The chassis was optimized by deleting FAR1 and SST2, respectively preventing PRP-induced cell cycle arrest and increasing sensitivity by reducing pathway deactivation. While these deletions are a common strategy ${ }^{7}$ their effects on heterologous signaling are poorly documented, so we measured how they influenced $p F I G 1:$ GFP response to alpha mating factor (Figure 1C). As expected, SST2 deletion increased sensitivity $(17.9 \mathrm{x})$ though background fluorescence also increased $(4.2 \mathrm{x})$, which limited fold induction of fluorescence. While Farl is not prescribed a role in pheromone sensitivity we found that FAR1 deletion decreased sensitivity both in wild type and sst 2 backgrounds (7.0x and $3.1 \mathrm{x}$ respectively). The background fluorescence of the sst 2 strain was also reduced by FAR 1 deletion from $4.2 \mathrm{x}$ to $1.6 \mathrm{x}$ that of wild type. This, together with the ability of a far 1 strain to facilitate longer assays, led us to select a sst 2 far 1 background even though the FAR1 deletion impacts sensitivity.

Next, the strain was engineered to produce cholesterol instead of ergosterol. Cholesterol and ergosterol are structurally similar, with zymosterol as the last common intermediate (Figure 1D). Following Souza et al., we deleted ERG5/6 and added zebrafish DHCR7/24 to block ergosterol production and redirect zymosterol to cholesterol ${ }^{22}$. In this modified cholesterol production pathway Erg2 and Erg3 fulfill the roles of human EBP and SC5DL respectively. GC-MS analysis showed 94\% of sterols were cholesterol with $4 \%$ dehydrolathosterol also present (Figure 1E; data not shown). These results indicate successful redirection of zymosterol to cholesterol.

Addition of yeast codon-optimized OPRM1, the gene encoding HsMOR, driven by the strong CCW12 promoter resulted in a candidate opioid biosensor. The sensitivity of this cholesterol producing biosensor, and a native ergosterol biosensor, was assessed by measuring fluorescence after 8 hours exposure to different concentrations of the HsMOR agonists [D-Ala ${ }^{2}, \mathrm{~N}-\mathrm{MePhe}^{4}$, Gly-ol]-enkephalin (DAMGO) and morphine. Importantly, initial tests indicated a strong $\mathrm{pH}$ dependence, with optimal morphine signaling at $\mathrm{pH} 7.1$, as opposed to the normal yeast growth media pH of 5-5.5 (Supplementary Figure 1B). We postulate that improved biosensor signaling at a $\mathrm{pH}$ of 7.1 results from conditions that more closely resemble the conditions HsMOR is exposed to in the brain ( $\mathrm{pH} 7.2$ intracellular ${ }^{29}, \mathrm{pH} 7.4$ extracellular $^{30}$ ).

With $\mathrm{pH}$ adjustment, both ergosterol and cholesterolproducing biosensors responded to DAMGO and morphine (Figure 1F). Consistent with the known cholesterol dependence of $\mathrm{HsMOR}^{9}$, the cholesterol-rich biosensor was dramatically more effective, with a lower $\mathrm{EC}_{50}(62 \pm 3 \mathrm{nM}$ vs $1.3 \pm 0.3 \mu \mathrm{M}$ DAMGO; $0.9 \pm 0.1 \mu \mathrm{M}$ vs $\sim 110 \pm 40 \mu \mathrm{M}$ morphine) and a larger proportion of cells signaling. The presence of any signaling in the ergosterol strain was unexpected given that previously [ $\left.{ }^{3} \mathrm{H}\right]$ DAMGO binding by HsMOR had not been detected in yeast ${ }^{9}$. The absence of binding may have been due to the use of a higher buffer $\mathrm{pH}$ (7.5) and/or lower receptor expression. Taken together, we constructed two opioid biosensors with different detection limits that demonstrate conversion of sterols to cholesterol can improve human GPCR function in yeast.

\section{An array of biosensors based on different opioid receptors reveals fidelity of agonist selectivity}

Next we expanded the set of receptors being tested to explore the degree of opioid receptor functional conservation in yeast. Opioid receptors exist throughout Vertebrata, from which a diverse set of receptors were selected, including five of each type: mu (MOR), kappa 4
(KOR) and delta (DOR). Within each type are receptors from humans (Homo sapiens, Hs), mice (Mus musculus, Mm), and zebrafish (Danio rerio, Dr). Additional receptors were included from the cow (Bos taurus, Bt), flying fox (Pteropus vampyrus, Pva), bearded dragon (Pogona vitticeps, Pvi), Burmese python (Python bivittatus, $\mathrm{Pb}$ ), and Mexican tetra, (Astyanax mexicanus, Am). As expected from the high degree of opioid receptor conservation, a MUSCLE-generated ${ }^{31}$ phylogenetic tree showed segregation by receptor type (Figure 2A). Furthermore, MORs and DORs clustered closely, consistent with the current model of MORs and DORs emerging from a common ancestral receptor ${ }^{32}$.

Biosensors based on these opioid receptors were tested for activity and agonist specificity. Agonists were selected based on human receptor specificities: morphine and met-enkephalin are broad-acting ${ }^{33}$, DAMGO and Endomorphins I/II are MOR-specific ${ }^{34,35}$, Deltorphin A and SNC80 are DOR-specific ${ }^{36,37}$, and Dynorphin A and Asimadoline are KOR-specific ${ }^{38,39}$. Most were short 4-17 residue peptides, or peptidebased, with the exception of the benzylisoquinoline alkaloid morphine and the two heterocycles SNC80 and Asimadoline. For each biosensoragonist pair a dose-response curve was made and $\mathrm{EC}_{50}$ was calculated (Figure 2B, Supplementary Figure 1/2). Remarkably, all strains showed some response to at least three of the agonists tested, and with the exception of the PvaMOR strain, all biosensors were sensitive enough to use agonist concentrations below $50 \mu \mathrm{M}$ to determine $\mathrm{EC}_{50}$.

Agonist specificities largely matched those of human receptors in endogenous conditions, though receptor sensitivity was reduced (Figure 2B/C). MORs and DORs responded to broad acting agonists morphine and met-enkephalin with $\mathrm{EC}_{50}$ of $30 \mathrm{nM}$ to $3 \mu \mathrm{M}$, while KORs responded poorly, consistent with KORs' reported low metenkephalin sensitivity but not their reported 47-538 nM morphine sensitivity ${ }^{33,34}$. MOR-specific agonists were detected by MORs with 60$500 \mathrm{nM} \mathrm{EC} \mathrm{C}_{50}$ while other receptor types were less sensitive $\left(\mathrm{EC}_{50}>5\right.$ $\mu \mathrm{M})$. Likewise, only DORs fully responded to the HsDOR-specific agonists deltorphin A and SNC80 (EC50S $2.5 \mathrm{nM}-4 \mu \mathrm{M})$. KOR-based biosensors were most sensitive to KOR-specific agonists with $\mathrm{EC}_{50} \mathrm{~S}$ as low as $6.3 \mathrm{nM}$, though most biosensors responded to dynorphin $\mathrm{A}$, consistent with reported MOR and DOR dynorphin A sensitivity ${ }^{34}$. While agonist specificities were maintained, biosensors displayed typespecific decreases in receptor sensitivity relative to values reported for more native environments, with DORs performing best (11x decrease) followed by KORs (43x decrease) and MORs (105x decrease) ${ }^{34-45}$ (Figure 2C).

The effects of an antagonist, naltrexone, were also determined ${ }^{34}$. Alone, naltrexone occasionally functioned as a partial agonist, at most eliciting a signaling population one seventh the size of that induced by a strong agonist (Figure 2B, Supplementary Figure 1). Antagonist activity was tested by incubating biosensors for eight hours with an amount of agonist sufficient for strong signaling $(2 \mu \mathrm{M}$ DAMGO, $0.1 \mu \mathrm{M}$ SNC80, $0.2 \mu \mathrm{M}$ asimadoline) and varying concentrations of naltrexone (Figure 2B, Supplementary Figure 3). Naltrexone blocked activity in all cases and, in line with binding coefficients previously determined in $\mathrm{CHO}$ cells $^{34}$, MORs were most sensitive ( $\left.\mathrm{IC}_{50}: 2-80 \mathrm{nM}\right)$, followed by KORs ( $\left.\mathrm{IC}_{50}: 0.8-500 \mathrm{nM}\right)$ and DORs (IC50: $2.5-3.2 \mu \mathrm{M}$ ). Together, the ability of these biosensors to reconstitute both agonist specificities and antagonist activity make them powerful tools for assessing how opioid receptors in native environments will respond to a compound. 
a

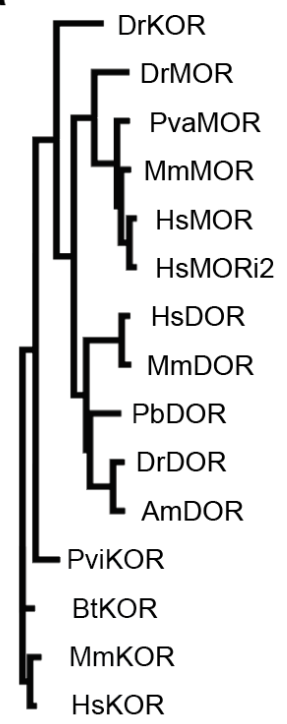

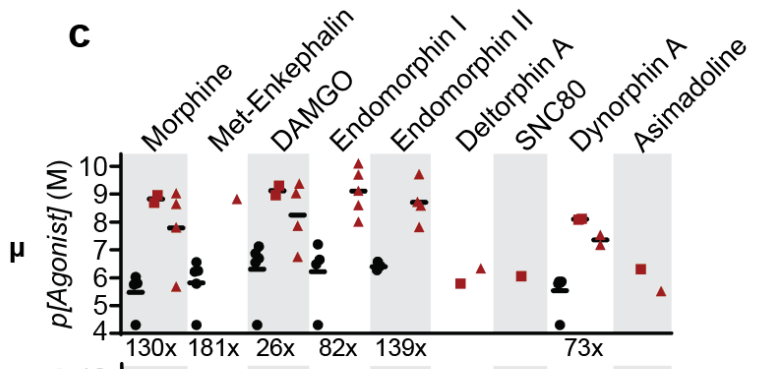

$\Delta$
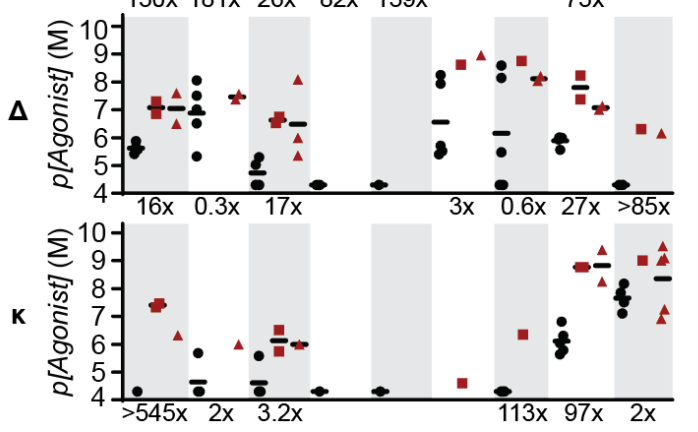

b

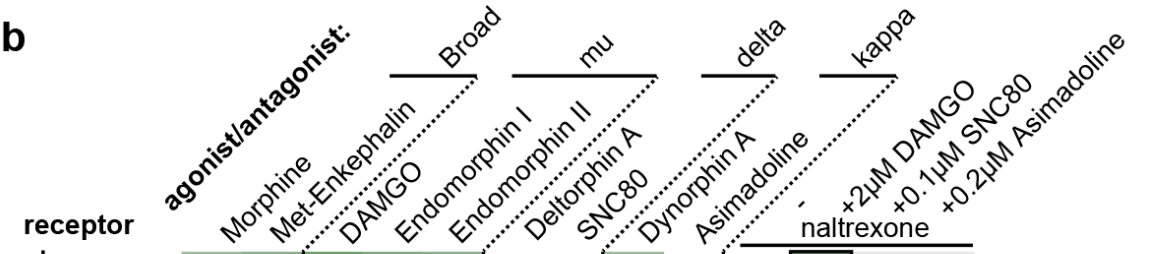

\begin{tabular}{|c|c|c|c|c|c|c|c|c|c|c|c|c|c|c|c|}
\hline \multicolumn{14}{|c|}{ 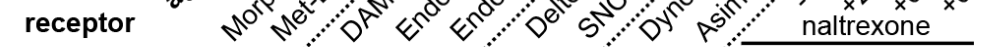 } & \multirow{3}{*}{$\begin{array}{r}p E C_{50} \\
(\mathrm{M})\end{array}$} & \multirow{3}{*}{$\begin{array}{l}\mathrm{EC}_{50} \\
\text { (nM) }\end{array}$} \\
\hline & HsMOR & 5.8 & 6.2 & 6.9 & 6.5 & 6.3 & _ & - & 5.8 & - & - & & & & \\
\hline & HsMORi2 & 5.8 & 6.2 & 6.7 & 6.4 & 6.3 & - & - & 5.8 & - & - & & & & \\
\hline & MmMOR & 6.0 & 6.6 & 7.1 & 6.6 & 6.4 & - & - & 5.9 & - & - & & & 9.5 & 0.3 \\
\hline & DrMOR & + & 5.8 & 6.5 & 7.2 & 6.6 & & - & 5.9 & & - & & & 9 & 1 \\
\hline & PvaMOR & - & + & + & + & - & - & - & + & - & - & & & 8.5 & 3.2 \\
\hline & HsDOR & 5.6 & 8.0 & 5.3 & + & - & 8.2 & 8.6 & 6.0 & + & - & & & 8 & 10 \\
\hline & MmDOR & 5.4 & 7.5 & 5.0 & + & - & 7.9 & 8.2 & 6.0 & + & 5.5 & & & 7.5 & 32 \\
\hline & DrDOR & 5.9 & 7.0 & + & + & + & 5.4 & + & 5.9 & - & + & & & 7 & 100 \\
\hline & $\mathrm{PbDOR}$ & - & 5.3 & - & - & - & 5.5 & 5.5 & 5.6 & - & - & & & 6.5 & 320 \\
\hline & AmDOR & 5.6 & 6.5 & + & + & - & 5.7 & + & 6.0 & - & - & & & 6 & 1000 \\
\hline & HsKOR & - & + & + & - & - & - & + & 6.3 & 7.9 & + & & 6.3 & 5.5 & 3200 \\
\hline \multirow{4}{*}{$\frac{0}{2}$} & MmKOR & + & + & + & - & - & - & + & 6.0 & 7.5 & + & & 6.0 & 5 & 10000 \\
\hline & BtKOR & - & - & - & - & - & - & - & 5.8 & 7.1 & - & & 6.9 & 4.5 & 32000 \\
\hline & DrKOR & - & + & + & - & - & - & - & 5.6 & - & - & & &,$+<4.3$ & $>50000$ \\
\hline & PviKOR & - & 5.7 & 5.6 & + & + & - & & 8 & 8.2 & & & 7.0 & & \\
\hline
\end{tabular}

Figure 2. Activity of opioid receptors in the cholesterol-producing strain. (a) A protein-based phylogenetic tree of opioid receptors selected to make biosensors. MOR, mu receptor, KOR, kappa receptor, DOR, delta receptor. Dr, D. rerio, zebrafish; Pva, P. vampyrus, flying fox; $\mathrm{Mm}, M$. musculus, mouse; $\mathrm{Pb}, P$. bivittatus, python; Am, A. mexicanus, Mexican tetra; Pvi, $P$. vitticeps, bearded dragon; Bt, B. taurus, cow. i2, isoform 2. (b) Activity of agonists and the antagonist naltrexone on opioid receptors assayed by flow cytometry. Agonists clustered by literature receptor-type specificity. $\mathrm{n}=3$, >2073 cells/condition/replicate. (c) Biosensor agonist sensitivities (black circles) relative to literature binding constants (red squares) and $\mathrm{EC}_{50} / \mathrm{IC}_{50}$ values (red triangles) ${ }^{34-45}$. Fold decrease in sensitivity of best biosensor relative to literature average is indicated.

\section{Signal sequences disrupt mu opioid receptor function}

Although the biosensors recapitulated the pattern of response seen in vertebrates, sensitivity was lower than in native cells, suggesting aspects of receptor expression or the signaling environment could be improved. To explore if opioid receptor sensitivity was limited by expression or localization defects, GFP-tagged HsMOR (HsMOR-GFP)

was imaged in a cholesterol-producing background. HsMOR-GFP primarily localized to the ER with a secondary vacuolar pool (Figure 3D). The unexpected lack of HsMOR-GFP on the plasma membrane, where functional GPCRs have previously been observed ${ }^{10}$, suggests a folding or trafficking defect may be leading to endoplasmic reticulum (ER) retention and/or misdirection to the vacuole. GFP tagging itself is unlikely to be causing mislocalization as the tag only reduced biosensor response to DAMGO by $34 \%$ (Supplementary Figure 1C). Given the degree of HsMOR-GFP ER retention, we reasoned that increasing plasma membrane localization might improve biosensor function.

GPCR expression and localization can be improved by appending N-terminal signal sequences $^{10,46}$, short peptides that mediate ER insertion $^{47}$. While integral membrane proteins, such as opioid receptors, often lack signal sequences because transmembrane helices are sufficient for ER targeting, adding the sequences can increase ER insertion speed, minimizing misfolding ${ }^{47,48}$. Therefore, the effects of appending signal sequences to HsMOR were assayed. We tested sequences from yeast $(\alpha$-mating factor prepro, $\alpha$ PrePro; Ost1 signal peptide - $\alpha$-mating factor pro, Ost1ss- $\alpha$ Pro) as well as others previously used to improve GPCR expression in mammalian cells (influenza Hemagglutinin; Restinin) ${ }^{46,49}$ (Figure $3 \mathrm{~A})$.

Appending signal sequences to HsMOR generally did not improve sensitivity to either DAMGO or morphine, and was instead disruptive in two distinct ways (Figure 3B/C). Only $\alpha$ PrePro increased sensitivity, by roughly two-fold for both agonists, whereas Ost1ss- $\alpha$ Pro was neutral and the Hemagglutinin and Restinin sequences caused 320 -fold decreases in sensitivity. While the yeast $\alpha$ PrePro and Ost1ss- $\alpha$ Pro sequences were neutral or beneficial for sensitivity, they dramatically decreased the maximum size of the signaling population, by $72 \%$ and $66 \%$ respectively. In contrast, the Hemagglutinin and Restinin sequences didn't significantly affect the DAMGOinduced signaling population, selectively disrupting the morphine response. Taken together, the signal sequence classes have contrasting effects: yeast-based sequences had a neutral or positive effect on sensitivity and a reduced signaling population, whereas the Hemagglutinin and Restinin sequences disrupted sensitivity but did not always reduce the signaling population.

To better understand this dichotomy, signal sequence-tagged HsMOR-GFP was imaged in a cholesterol-producing background (Figure 3D). Strikingly, the $\alpha$ PrePro and Ost1ss- $\alpha$ Pro sequences resulted in enlarged granular cells, expanded ER membranes and relocalization 
a aPrePro

MRFPSIFTAVLFAASSALA-APV(+61)KREA-

Ost1ss- $\alpha$ Pro

MRQVWFSWIVGFLCFFNVSSA-APV(+61)KREAEA-

Hemagglutinin

MKTIIALSYIFCLVFA-

b

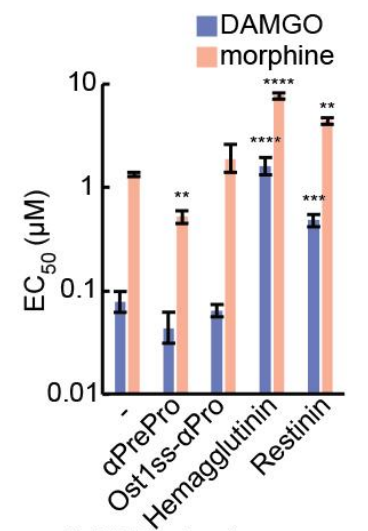

HsMOR signal sequence

$\mathrm{Hs}$

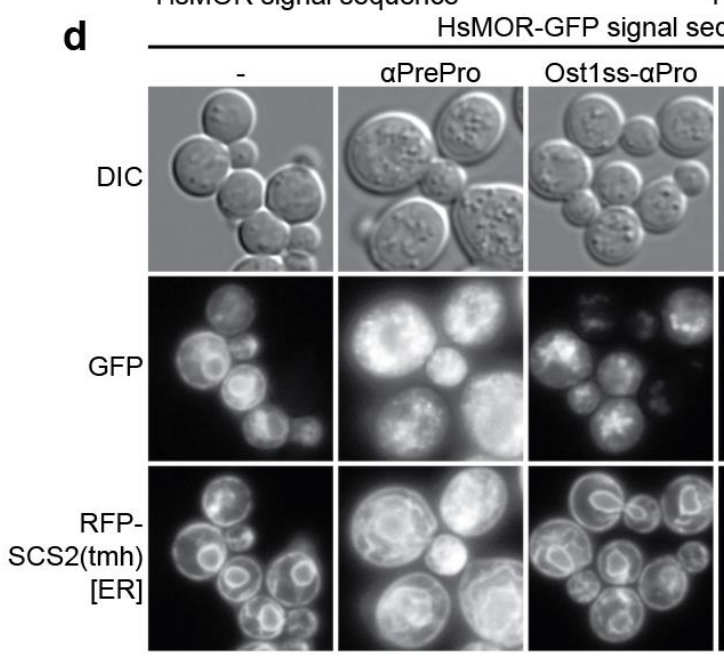

Restinin

MKALCLLLLPVLGLLVSSA-

C

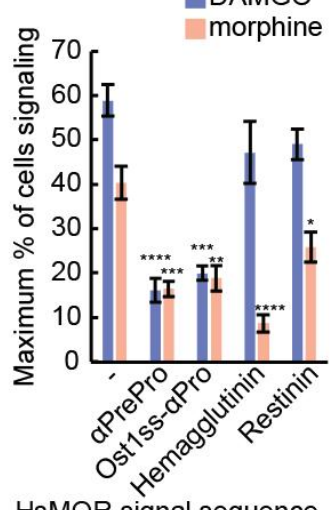

HsMOR signal sequence equence

decreased the signaling population such that the signal sequences were not beneficial.

\section{Biosensors recapitulate the effects of missense mutants in HsMOR}

Our biosensor platform may enable convenient characterization of rare opioid receptor alleles. Introduction of receptor variants should allow measurement of altered receptor sensitivities and signaling strength, potentially predicting clinically relevant changes in responses to analgesics. To probe our platform's ability to detect these changes we tested known HsMOR missense mutations (Figure 4A).

Variant HsMORs were introduced into the biosensor background and response to DAMGO and morphine was measured (Figure 4B/C). In agreement with previous work, the signal transduction-defective HsMOR(R181C) mutant was unable to respond to either DAMGO or morphine ${ }^{50-52}$. While dramatic defects were clearly detected, alleles associated with subtle defects were also explored. Previous descriptions of the relatively common (8-16\% frequency) HsMOR(N40D) allele are more ambiguous, alternately describing no effect on agonist affinities or decreased $\beta$-endorphin affinity, while decreased analgesic response to morphine has also been reported ${ }^{50,53}$. In our biosensor, the $\mathrm{N} 40 \mathrm{D}$ variant did not differ in DAMGO response though it displayed a decrease in morphine sensitivity $\left(\mathrm{EC}_{50}+38 \%\right)$, consistent with the reported decrease in morphine-based analgesia. Another variant, S268P, has a disrupted phosphorylation site and has been associated with reduced $\mathrm{G}$ protein coupling and reduced internalization and desensitization. A HsMOR(S268P)-based biosensor displayed decreased sensitivity to DAMGO $\left(\mathrm{EC}_{50}+84 \%\right)$ and morphine $\left(\mathrm{EC}_{50}\right.$ $+75 \%$ ), consistent with diminished $\mathrm{G}$ protein coupling and raising the possibility of native yeast kinases acting on exogenous GPCRs.

Ravindranathan et al. characterized other

Figure 3. Effect of signal sequences on HsMOR function. (a) N-terminal signal sequences tested on HsMOR. (b) Effect of signal sequences on HsMOR biosensor sensitivity to DAMGO and morphine. Unpaired one-way ANOVA of pEC50s: $\mathrm{n}=3,>7595$ cells/strain/replicate; $\mathrm{P}<0.0001$ for each agonist; Dunnett's multiple comparisons tests against WT shown. (c) Effect of signal sequences on HsMOR signaling population. Unpaired one-way ANOVA: $n=3$, $>7595$ cells/strain/replicate; $\mathrm{P}<0.0001$ (DAMGO) and $\mathrm{P}<0.001$ (morphine); Dunnett's multiple comparisons tests against WT shown. (d) Imaging of HsMOR-GFP with the indicated signal sequences in a cholesterol-rich background with the ER marker RFP-SCS2(tmh). Error bars indicate SEM. Scale bar is $2 \mu \mathrm{m} .{ }^{*}, \mathrm{P}<0.05 ;{ }^{* *}, \mathrm{P}<0.01 ;{ }^{* * *}, \mathrm{P}<0.001 ;{ }^{* * * *}, \mathrm{P}<0.0001$.

of HsMOR-GFP to puncta. In contrast, the Hemagglutinin and Restinin tags did not disrupt cellular morphology and HsMOR-GFP remained ER-localized, though the vacuolar pool may have increased. These results suggest that the yeast-based sequences cause global cellular disruptions, perhaps through partial HsMOR-GFP misfolding, which may be associated with premature ER exit. Cellular stress likely disrupts signaling, leading to the observed reductions in biosensor signaling competency. The other sequences did not disrupt cellular morphology and consequently did not display consistent decreases in the biosensor signaling population. The link between cellular localization and sensitivity was unclear. Overall, while the yeast signal sequences subtly improved HsMOR sensitivity, the associated cellular disruptions
HsMOR variants that resulted in mild decreases (S42T, C192F) or an increase (S147C) in sensitivity to DAMGO and morphine ${ }^{52}$. Correspondingly, a $\operatorname{HsMOR}(\mathrm{S} 42 \mathrm{~T})-$ based biosensor displayed decreased signaling populations with both agonists, and a HsMOR(C192F)based biosensor had significantly lower sensitivities to DAMGO $\left(\mathrm{EC}_{50}+37 \%\right)$ and morphine $\left(\mathrm{EC}_{50}+125 \%\right)$. However, HsMOR(S147C) did not show improved sensitivity, instead resulting in a mild $10 \%$ decrease in the DAMGO-induced signalling population. Thus, the HsMOR biosensor provides a powerful platform to screen variants for changes in activity, which could inform how patients will respond to opioid-based analgesics.

\section{Exploring the functional significance of HsMOR terminal domains}

We further applied our platform to explore how additional HsMOR structural variants affect receptor activity and localization in yeast. Opioid receptor terminal domains are moderately conserved, often containing trafficking motifs, glycosylation sites and 
a

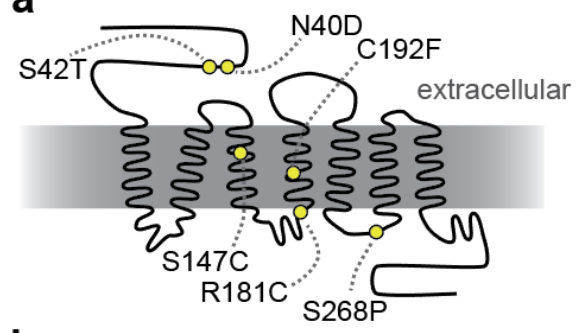

b
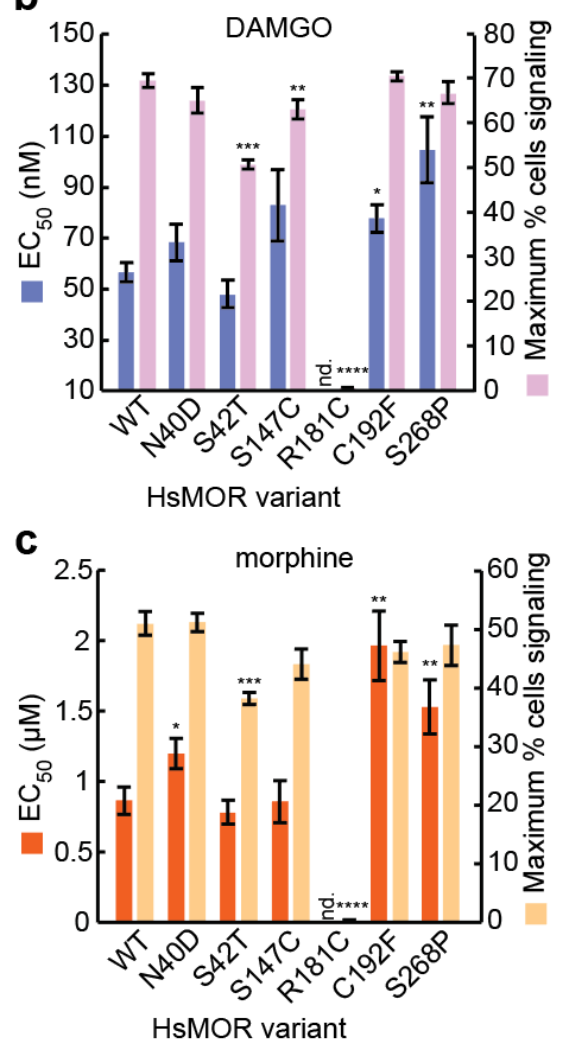

Figure 4. The effects of HsMOR missense mutations on opioid biosensors activity. (a) HsMOR snake plot with previously identified missense mutations. Biosensors based on these mutants were assayed for DAMGO response (b), or morphine response (c), by flow cytometry. Paired one-way ANOVAs of DAMGO $p E C_{50} \mathrm{~S}$ and maximum percentage of cells signaling: $n=6,>2956$ cells/condition/replicate; $P=$ 0.0002 and $P<0.0001$ respectively; Dunnett's tests against WT shown. Paired one-way ANOVAs of morphine $p E C_{50}$ S and maximum percentage of cells signaling: $n=6,>4761$ cells/condition/replicate; $\mathrm{P}=0.0033$ and $\mathrm{P}<0.0001$ respectively; Dunnett's tests against WT shown. Error bars indicate SEM. * $\mathrm{P}<0.05 ;{ }^{* *}, \mathrm{P}<0.01$; ${ }^{* * *}, \mathrm{P}<0.001 ;{ }^{* * *}, \mathrm{P}$ $<0.0001$.

phosphorylation sites, collectively contributing to folding, localization and modification of activity ${ }^{32,54}$. We first made variants lacking putative trafficking motifs $\mathrm{R}_{367 \mathrm{xR}}$ and $\mathrm{L}_{389} \mathrm{XxLE}$, or all five putative N-linked glycosylation sites (Figure 5A). RxR motifs can bind the coatomer protein I (COPI) complex and have been shown to mediate delta opioid receptor ER/Golgi retention ${ }^{55}$, while LxxLE can be recognized by COPII, facilitating ER exit ${ }^{56}$. N-glycosylation aids in protein quality control and contributes to DOR and KOR folding, stability and trafficking ${ }^{57-59}$. In response to DAMGO and morphine biosensors based on all variants displayed subtle decreases in sensitivity (1.6-2.6-fold), suggesting these regions do not greatly contribute to folding or trafficking of HsMOR in yeast (Figure 5B). Consistently, isoform 2 of HsMOR, which contains a LENLEAETAPLP>VRSL C-terminal substitution and therefore lacks the LxxLE motif has a similar signaling profile to isoform 1 (Figure 2B). However, removal of the RxR motif and the N-glycosylation sites did decrease the percent of cells signaling by up to $40 \%$ and $28 \%$ respectively, highlighting their contribution to achieving optimal activity (Figure 5C). In line with the overall mild defects, GFP-tagged variants displayed wild type localization (Figure 5D).

Next we tested complete removal of the HsMOR N- and Cterminal domains as well as substitution of these domains with those of the endogenous GPCR Ste2, as a small Ste2 N-terminal swap previously improved exogenous GPCR activity ${ }^{6}$. N-terminal deletion decreased DAMGO and morphine sensitivity by 6.8 - and 4.6 -fold respectively, in line with a previous report of a similar deletion causing a 3.3-fold drop in DAMGO affinity in HEK 293 cells $^{60}$ (Figure 5B). Thus, the moderate functional contribution of the N-terminus appears conserved. In contrast with previous Ste2 swaps, complete substitution of the HsMOR $\mathrm{N}$-terminus with that of Ste2 also decreased receptor function, reducing HsMOR DAMGO sensitivity 30-fold and decreasing the morphine signaling population by $72 \%$ (Figure $5 \mathrm{~B} / \mathrm{C}$ ). However, unlike the $\mathrm{N}$ terminal deletion, which displayed aberrant localization to ERassociated puncta, the $\mathrm{N}$-terminal substitution displayed a wild type localization (Figure 5D). This suggests the Ste2 N-terminus is sufficient for maintaining localization and that localization poorly correlates with function.

C-terminal domain deletions or Ste2 substitutions also displayed a disconnect between localization and function as they showed no activity while maintaining nearly wild type localization, though with increased vacuolar pools (Figure 5B/C/D). The failure of the C-terminal mutants to signal was unexpected as a similar C-terminal deletion displayed only a small reduction in DAMGO sensitivity when expressed in $\mathrm{CHO}_{\text {cells }}{ }^{61}$. While this may indicate more stringent requirements for activity in yeast, the C-terminal deletions used here disrupt a short cytosolic helix (helix 8) next to the transmembrane domain that, while not involved directly in $\mathrm{G}$ protein binding or signal transduction, may contribute to the functional conformation of the receptor ${ }^{62,63}$. Taken together our results show our biosensors can be used to assess how domains and motifs contribute to function, and highlight the difficulty in linking activity to localization.

\section{Modifying membrane sterols alters HsMOR biosensor function}

Cholesterol biosynthetic intermediates are typically present in plasma membranes at low concentrations, and accumulations are linked to developmental and neurological defects ${ }^{64}$. However, it is unclear to what extent these intermediates can fulfill the roles of cholesterol in promoting GPCR activity. Profiles of sterol intermediates may exist that further promote GPCR signaling in yeast without cholesterol-associated growth and transformation defects ${ }^{22}$.

To search for sterol intermediate profiles that could improve the performance of the HsMOR-based biosensor, we adjusted the expression of human cholesterol biosynthesis genes DHCR24, EBP, SC5DL and DHCR7 in an erg2/3/5/6 $\Delta$ background (Figure 1D). While use of the zebrafish DHCR7 and DHCR24 enzymes effectively produced cholesterol, we tested if varying expression of the human enzymes could lead to a more functional sterol environment. The human genes were introduced to the ergosterol biosynthesis gene loci, driven by the native 
bioRxiv preprint doi: https://doi.org/10.1101/2021.05.12.443385; this version posted May 14, 2021. The copyright holder for this preprint (which was not certified by peer review) is the author/funder. All rights reserved. No reuse allowed without permission.

a

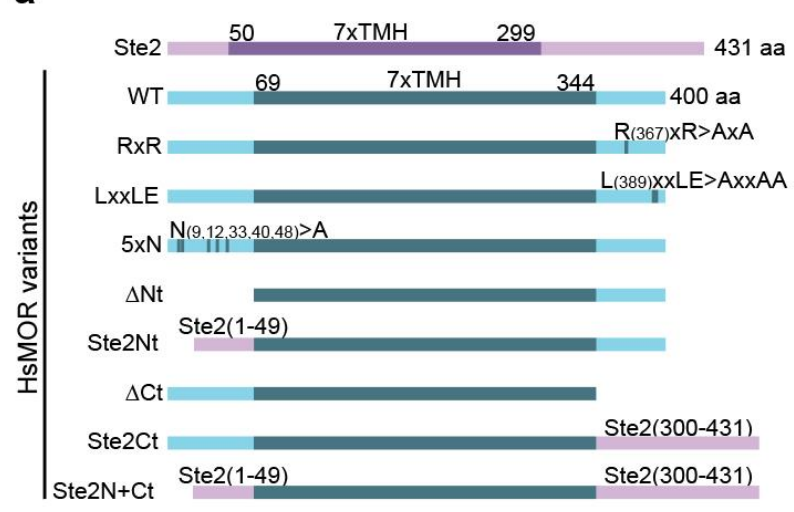

b

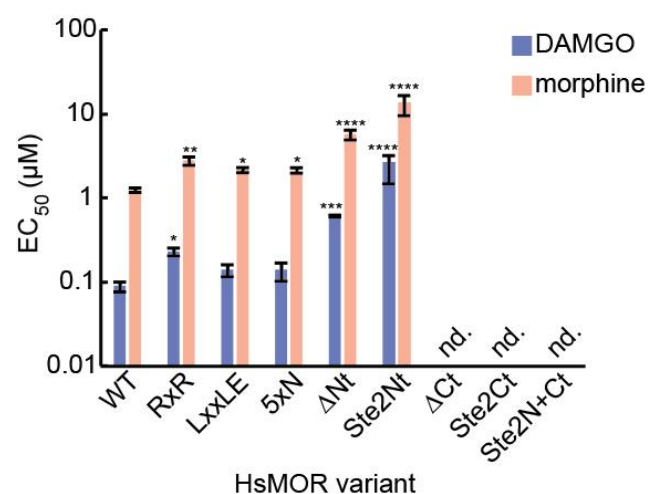

C

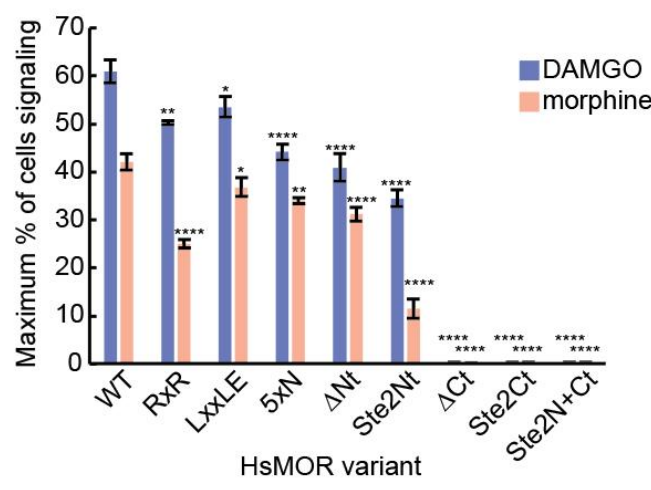

d
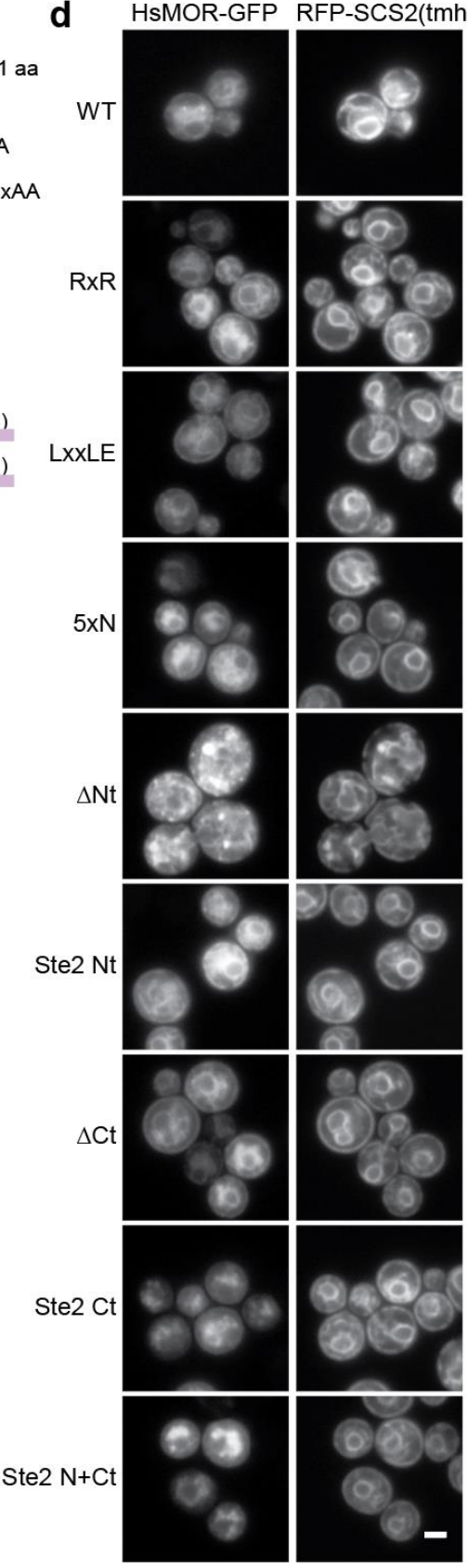

Figure 5. The functional requirements of HsMOR $\mathrm{N}$ - and $\mathrm{C}$-terminal domains on biosensor activity. (a) Domains of Ste2, HsMOR and HsMOR variants with the conserved seven transmembrane helix $(7 \times \mathrm{TMH})$ domains indicated. (b) DAMGO and morphine sensitivity of biosensors based on the indicated HsMOR variants. Unpaired one-way ANOVA of $p E C_{50 \mathrm{~S}}: \mathrm{n}=3,>7464$ cells/strain/replicate; $\mathrm{P}<0.0001$ for both agonists; Dunnett's tests against WT shown. (c) The effect of $\mathrm{N}$ - and $\mathrm{C}$-terminal HsMOR mutations on the population of signaling biosensor cells. Unpaired one-way ANOVA: $n=3$, $>7464$ cells/strain/replicate; $P<0.0001$ for both agonists; Dunnett's tests against WT shown. (d) Imaging of C-terminally GFP-tagged HsMOR variants in a cholesterolproducing background with ER marker RFP-SCS2(tmh). Error bars indicate SEM. Scale bar is $2 \mu \mathrm{m} .{ }^{*}, \mathrm{P}<0.05 ;{ }^{* *}, \mathrm{P}<0.01 ;{ }^{* *}, \mathrm{P}<0.001 ;{ }^{* * *}, \mathrm{P}<0.0001$.

yeast promoters (erg5 $4:: H s . D H C R 24, \quad \operatorname{erg} 6 \triangle:: H s . D H C R 7$, $\operatorname{erg} 3 \Delta:: H s . S C 5 D L, \operatorname{erg} 2 \Delta:: H s . E B P)$. GC-MS analysis of sterols showed this strain contained the intermediates zymosterol, dehydrolathosterol, and 7-dehydrodesmosterol, while products of DHCR7 and DHCR24 activity failed to accumulate (Figure 6B/C).
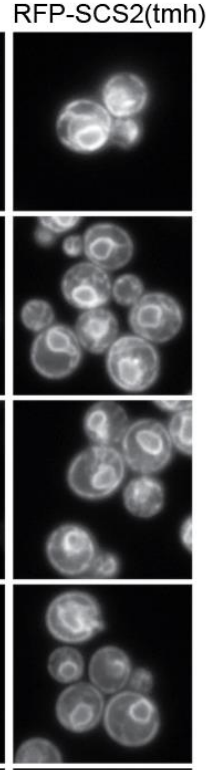

Hypothesizing that the ergosterol promoters were insufficient for the potentially low activity of the heterologous enzymes, we genomically integrated cassettes containing additional copies of the cholesterol biosynthetic genes under high, medium, or low strength yeast promoters to generate strains with modified sterol profiles (Figure 6A). Of the 256 combinations, 249 were successfully constructed and assayed for response to $10 \mu \mathrm{M}$ and $1 \mu \mathrm{M}$ DAMGO, the concentrations roughly required to reach the $\mathrm{E}_{\max }$ and $\mathrm{EC}_{50}$ in the wild type background (Figure 1F). Responses ranged from $21 \%-61 \%$ and $8 \%-47 \%$ of cells signaling at $10 \mu \mathrm{M}$ and $1 \mu \mathrm{M}$ DAMGO respectively. HsDHCR7 was inactive, confirmed by failure of a Wilcoxon signed-rank test $(\mathrm{P}>0.05)$ comparing the strong DHCR7 expression and no gene conditions, and the absence of products from DHCR7 activity in downstream sterol analyses (Supplementary Figure 3A, data not shown). Therefore, we excluded DHCR7 from our analysis and selected $39 \%$ of strains from this collapsed set for membrane sterol composition analysis (Supplementary Figure 3A). Determination of sterol composition by GC-MS revealed that most variation was in 7-dehydrocholesterol, zymosterol, zymostenol, and lathosterol (Figure 6C). Additionally, dose responses using the agonist DAMGO were performed in tandem, and $\mathrm{EC}_{50}$ values for each strain were determined (Figure 6C).

Hierarchical clustering identified trends in the composition of sterol intermediates; in particular variations in DHCR24 promoter strength led to the largest changes in sterol composition, with higher promoter strength correlating with decreased HsMOR sensitivity (Figure 6C). The single copy of DHCR24 in the base strain proved insufficient to produce zymostenol, lathosterol, and 7-dehydrocholesterol. Accordingly, the presence of these intermediates correlate with higher $\mathrm{EC}_{50}$ values. The cholesterol-producing biosensor strain proved most sensitive with an $\mathrm{EC}_{50}$ approximately four times lower than the most sensitive strain identified from the screen (Figure 6C).

We then performed a linear regression analysis on the sterol intermediate percentages and $\mathrm{EC}_{50} \mathrm{~S}$ to establish the relationship between sterol composition and signaling. Sterol composition and $\mathrm{EC}_{50} \mathrm{~S}$ were found to be highly correlated $\left(\mathrm{R}^{2}=0.84\right)$, with cholesterol and ergosterol composition indicative of low and high values respectively (Supplementary Figure 3D). Taken together, varying the cholesterol biosynthesis intermediates modulated HsMOR biosensor 

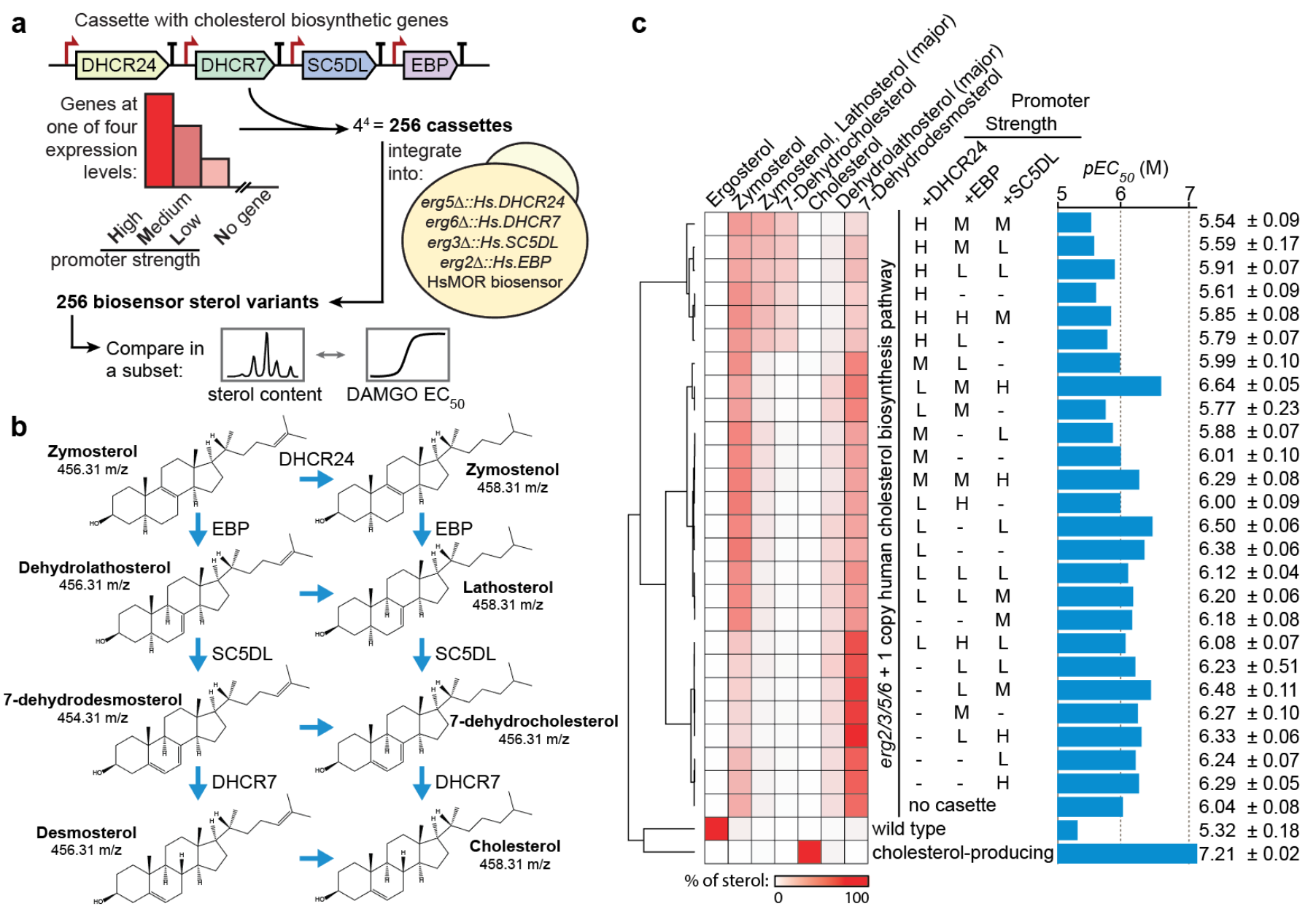

Figure 6. The effect of membrane sterol composition on opioid biosensor signaling efficiency. (a) Construction of an array of putative biosensor strains with systematically varied promoter strength for the cholesterol biosynthetic genes downstream of zymosterol. Cassettes containing these genes under different promoters were integrated into an HsMOR biosensor background with an additional copy of these enzymes in place of the final ergosterol biosynthetic genes (ERG2/3/5/6). (b) Cholesterol biosynthesis intermediates after zymosterol. (c) Sterol analysis and full dose responses with DAMGO of $39 \%$ of biosensors from the collapsed screen. Percentages of total sterol content for the intermediates were determined and $p E C_{50}$ concentrations were calculated.

sensitivity, but the cholesterol-producing background remained most sensitive.

\section{Cholesterol improves human class A GPCR function in yeast}

To explore how broadly cholesterol improves functional expression of human GPCRs in yeast, we introduced seven different GPCRs into wild type and cholesterol-producing biosensor backgrounds. These receptors belong to three GPCR classes, all can couple with the $\mathrm{G}_{\mathrm{i} / \mathrm{o}}$ chimera, and four of them, HTR4B, GLP1R, SSTR5, and FPR1, have been shown to function in yeast ${ }^{65-68}$ (Figure 7A). Of the resulting putative biosensors, all strains with class A receptors showed response to agonists at $10 \mu \mathrm{M}$ and lower, whereas no class $\mathrm{B}$ or $\mathrm{C}$ receptors signaled in either sterol background (Figure $7 \mathrm{~B}$ ). Of the receptors reported to be active in yeast, only GLP1R failed to signal, possibly due to the use of different assays.

We generated dose-response curves for the active receptors, FPR1, SSTR5, HTR4B in both sterol backgrounds; a dose response could not be measured NPY1R since it only responded to neuropeptide $\mathrm{Y}$ concentrations approaching $10 \mu \mathrm{M}$ (Figure 7B). Remarkably, sensitivities of all biosensors were greater in the cholesterol background, with reductions in EC50 ranging from 3-fold (SSTR5, HTR4B) to 47-fold
(FPR1). Likewise, the maximum percent of cells signaling increased in all cases, including NPY1R biosensor (1.2 to 2-fold). Our results strongly suggest that the activity of at least Class A GPCRs can often be improved by expression in a cholesterol-producing background.

\section{DISCUSSION}

We have shown that engineering yeast to produce cholesterol is an effective strategy for improving vertebrate GPCR activity in yeast, thereby enabling the generation of opioid biosensors with nanomolar sensitivities and expected agonist selectivities. This allowed us to evaluate the structural requirements for HsMOR function in yeast and recapitulate many defects associated with clinically relevant missense mutations. Systematic modification of the sterol biosynthetic pathway revealed that while the presence of upstream cholesterol intermediates can improve activity, a cholesterol producing background is most effective for HsMOR function. The presence of cholesterol also improved the function of several other GPCRs (FPR1, HTR4B, SSTR5 and NPY1R) indicating that modification of sterols is a general tool for the functional expression of animal GPCRs in yeast.

GPCRs can require cholesterol for normal function or regulation, likely due to both specific GPCR-cholesterol interactions ${ }^{20}$ 
a

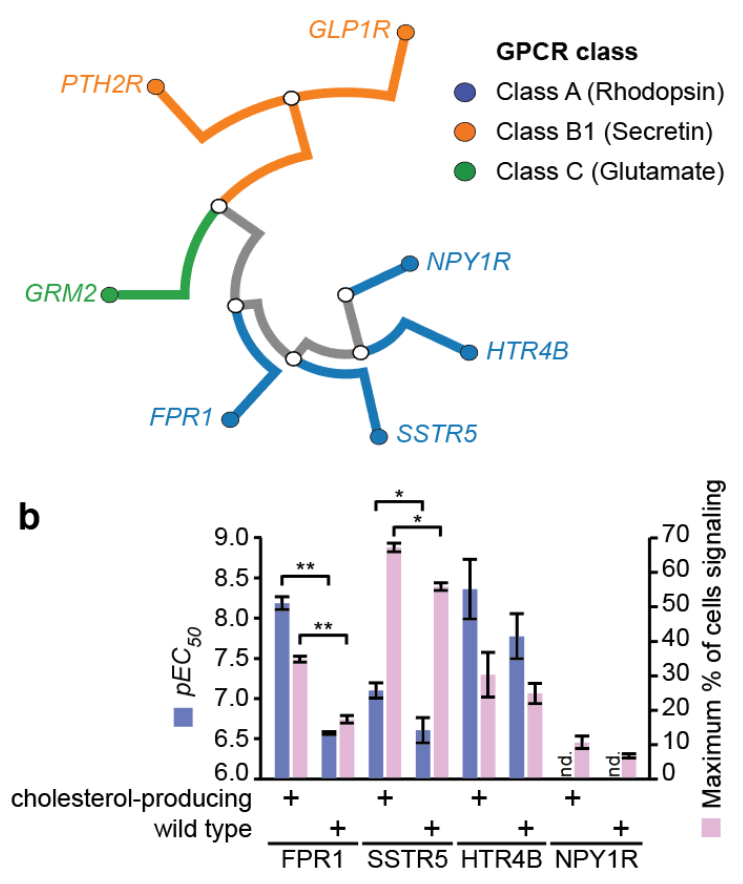

Figure 7. Effect of cholesterol on the activity of a broad range of human GPCRs. (a) Phylogenetic relationships of human GPCRs tested. (b) GPCRs' response to neuropeptide Y (NPY1R), Serotonin (HTR4B), Somatostatin-14 (SSTR5), Nformyl-Met-Leu-Phe (FPR1), L-glutamate (GRM2), parathyroid hormone $(P T H 2 R)$ and glucagon-like peptide-1 (GLP1R) in wild type and cholesterol-producing biosensor backgrounds measured by flow cytometry. FAR1 is present in the cholesterol-producing background as it facilitated strain construction. $p E C_{50 S}$ and maximum signaling population of the four biosensors showing activity at or below $10 \mu \mathrm{M}$ agonist. Paired two-tailed t test, $n=3,>7464$ cells/strain/replicate. Error bars indicate SEM. ${ }^{*}, \mathrm{P}<0.05 ;{ }^{* *}, \mathrm{P}<0.01$.

and non-specific effects such as increased membrane fluidity or the facilitation of lipid subdomains ${ }^{69}$. By comparing GPCR activity in cholesterol- and ergosterol-producing yeast we indirectly assessed the extent to which cholesterol is specifically required for human GPCR activity. Remarkably, cholesterol increased the sensitivity of all tested GPCRs, even though only HsMOR has reported cholesteroldependence. This suggests that cholesterol often improves human GPCR function beyond a non-specific requirement for sterols in the membrane. Conversely, non-native sterols may actively disrupt function, as Lagane et al. could detect DAMGO binding by HsMOR in yeast lysates only after ergosterol depletion with methyl- $\beta$-cyclodextrin 9 This effect likely contributed to the performance improvements of biosensors producing sterol intermediates. Taken together, the frequency with which GPCRs have evolved to utilize direct interactions with native sterols may be underestimated.

Though many GPCRs benefited from the presence of cholesterol, HsMOR and FPR1 displayed the greatest improvements in sensitivity (Figures 1 and 7). HsMOR cholesterol dependence was expected as cholesterol is bound in HsMOR crystal structures ${ }^{62}$ and there is evidence that cholesterol directly promotes an active conformation ${ }^{70}$, partitions the receptor into more functional subdomains ${ }^{71}$, and aids in dimerization $^{72}$. FPR1 has not been shown to interact with cholesterol, but a crystal structure of the closely related GPCR FPR2 (69\% identity) contains many associated cholesterol molecules ${ }^{73}$. Though there is evidence that both receptors could be directly interacting with cholesterol in yeast, the degree to which this is occurring and the mechanism by which this improves activity remains to be resolved. The mild cholesterol dependence of the other receptors likely reflects a more limited potential for cholesterol binding. While cholesterol often improves activity, it has been shown to disrupt the activity of some receptors including the $M_{2}$ muscarinic acetylcholine receptor ${ }^{74}$, type 1 cannabinoid receptors ${ }^{75}$, and rhodopsin ${ }^{76}$. It remains to be determined if the activity of these receptors is similarly disrupted in a cholesterolproducing yeast strain and if rules can be developed to predict which receptors will most benefit from conversion of ergosterol to cholesterol.

Small differences in sterol structure appear to have significant effects on HsMOR signaling. Screening HsMOR-based biosensors producing different sterol intermediates revealed that any combination of cholesterol intermediates increases sensitivity relative to ergosterol, though 7-dehydrocholesterol, lathosterol, and zymostenol were least beneficial. These three intermediates all require the DHCR24-mediated reduction of the alkene at $\mathrm{C} 24$, suggesting that this reduction without the rearrangement of the double bond within the rings is most disruptive to HsMOR function. While no strain producing sterol intermediates promoted HsMOR activity as much as the cholesterol-producing strain, all were effective. This was surprising given that in humans enrichment of these intermediates, including zymosterol, lathosterol and 7dehydrocholesterol, is disruptive and linked to several diseases ${ }^{64}$. Indeed, one GPCR, HTR1A, can be disrupted by increasing 7dehydrocholesterol levels to mimic Smith-Lemli-Opitz Syndrome ${ }^{77}$. Our sterol intermediate biosensors offer surrogate strategies to screen for other similarly disrupted GPCRs.

Our cholesterol-rich background enabled all opioid receptors to signal, generally with expected agonist specificities, allowing us to establish interspecies conservation of receptor function. The mammalian opioid receptors consistently displayed specificities similar to those of humans, whereas the responses of less related receptors were more variable. Some receptors such as the flying bat MOR, python DOR, or the zebrafish KOR only weakly responded to some of the agonists. In contrast, the bearded dragon KOR had the strongest response to KOR agonists and was also able to respond to the MOR-specific agonist DAMGO. The zebrafish DOR and KOR-based biosensors each showed no response to one of the two type-specific agonists tested, in line with previous work indicating the zebrafish DOR responds more strongly to general agonists than MOR, KOR, or DOR-specific agonists ${ }^{78}$. Indeed, a previous model suggests that there is an increased rate of divergence of mammalian opioid receptors from ancestral receptors relative to those of fish and reptiles, leading to more robust agonist specificities ${ }^{32}$. While our data partially supports this model, we find agonist specificities to be widely conserved.

While specificity was well conserved, opioid biosensors were on average 54-fold less sensitive than values previously determined for receptors in more native environments (Figure 2C). Only the HsDOR met-enkephalin response outperformed reported sensitivities with an $\mathrm{EC}_{50}$ of $10 \mathrm{nM}$, a 3-fold improvement. Notably, the drop in sensitivity of MORs in our biosensors was largest and roughly ten times greater than that of DORs, a substantial difference given their close evolutionary and structural relationships (Figure 2A). Perhaps MORs, which appear to 
have the highest evolutionary rate $^{32}$, diverged to require additional features of the vertebrate environment for full function. Species of origin was poorly correlated with sensitivity as the origins of the most sensitive mu, delta, and kappa receptors were diverse: mice, humans, and bearded dragons respectively. This indicates that opioid receptor sensitivity may be heavily influenced by sporadic mutations that coincidentally improve performance in yeast.

Thus, there is room to improve opioid biosensor performance, perhaps by further adjusting the biosensor environment or its components. Here, GPCRs were generally codon optimized to improve yeast expression. However, additional tests on a subset of six opioid biosensors, two of each receptor type, found that native genes improve sensitivity by as much as 31-fold for the SNC80 response of PbDOR, and 3.9-fold on average (Supplementary Figure 1). Though the sensitivity improvement was tempered by a 1.4-fold reduction in percent of cells signaling, using GPCRs with native codons may be beneficial overall. This may be because native genes contain rare codons, decreasing the rate of translation, which could promote opioid receptors achieving optimal folds. Other approaches to improve biosensor activity may include strengthening the link to the pheromone response pathway, adding potential chaperones, or performing unbiased screens for yeast deletions that improve activity. Introducing enzymes responsible for post-translational modifications such as palmitoylation ${ }^{54}$ or attempting to adjust yeast membrane thickness ${ }^{79}$ may also be helpful. Alternatively, applying slower biosensor outputs that allow greater signal accumulation, such as the $24 \mathrm{~h} \beta$-galactosidase used by Olesnicky et al., could improve sensitivity ${ }^{25}$.

Our opioid biosensors and sterol-modified biosensor backgrounds have many applications. The speed and low cost of using our opioid biosensors for screening compounds for receptor typespecific activation should make them an attractive tool to bridge computational docking studies ${ }^{80}$ and more costly screens in human cell lines based on protein complementation ${ }^{40}$ or bioluminescence resonance energy transfer ${ }^{81}$. Currently our opioid biosensors are unable to measure modes of signaling beyond $G$ protein activation, such as $\beta$-arrestin recruitment, which is thought to cause many of the side effects of opioids. This makes the biosensors less useful for drug discovery efforts which are focused on identifying compounds that display biased agonism towards $\mathrm{G}$ protein activation. However, our biosensors are compatible with the PRESTO-Tango ${ }^{82}$ system for detecting GPCR- $\beta$ arrestin interactions, which would allow future biosensors to detect biased agonism. By increasing throughput of production assays from hundreds to thousands, these biosensors will also aid in the ongoing development of opiate production strains ${ }^{83}$. Furthermore, it may be possible to adapt the opioid biosensors to field tests for opioid detection. Colorimetric assays based on yeast biosensors have been reported previously ${ }^{84}$, and in principle our biosensors could be used to test a sample for the degree of opioid activity independent of identifying the compounds present. This may enable testing kits that could be used to assess the amount of a sample likely to cause an overdose. Beyond opioid biosensors, our sterol modified platform should enable the expression of many other human GPCRs in yeast, generating an array of new biosensors and tools for the deorphanization of GPCRs.

\section{MATERIALS AND METHODS Strains and Plasmids}

Strains and plasmids are listed in Supplementary Table 1 and 2. Strains were derived from BY $4741^{85}$ using CRISPR-Cas9 as follows. A Cas9 (CEN6 URA3) vector was constructed using components of the Yeast Toolkit ${ }^{86}$, with $p P G K 1$-Cas9-tENO2 and up to four sgRNAs expressed from a tRNA ${ }^{\text {Phe }}$ promoter with a 5' HDV ribozyme site and a SNR52 terminator. Alternatively a Cas9 vector derived from the vector described in Ryan et al. was used ${ }^{87}$. Strains were constructed by transforming yeast with a Cas 9 vector, unique protospacers guiding Cas9, and a double stranded repair template introducing deletions or modifications. Deletions and modifications were confirmed by colony PCR and sequencing respectively. All protospacers and repair template sequences are listed in Supplementary Table 3. Yeast were transformed using either the Zymo Research EZ Yeast Transformation II Kit (cat. T2001) or a modified Gietz protoco ${ }^{88}$.

Plasmids were constructed using Golden Gate assembly ${ }^{89}$ of components from the Yeast Toolkit ${ }^{86}$ and elsewhere. Opioid receptors were all expressed from the same $2 \mu$ HIS 3 backbone assembly (ConLS'CCW12p-GPCR-SSA1t-ConRE'-HIS3-2 $\mu$-KanR-ColE1), while FPR1 was on a similar vector with a TDH1 terminator and other GPCRs were expressed from a ConLS'-CCW12p-GPCR-SSA1t-ConRE'-URA3-2 $\mu$ KanR-p15a backbone. GPCRs were ordered as either gblocks from IDT or clonal genes from Twist Biosciences. DNA sequences used are listed in Supplementary Table 4 and GPCRs were yeast codon optimized unless specified as non-Codon Optimized (nCO).

\section{Sterol extraction}

Yeast strains were grown to either mid-log (8hrs) or saturation (48 hrs) from single colonies. Since the growth rates of these strains were different, wet weights were adjusted to $50 \mathrm{mg}$ and $150 \mathrm{mg}$ for the $8 \mathrm{hr}$ and $48 \mathrm{hr}$ timepoints respectively. These were then suspended in glass tubes containing $3 \mathrm{ml}$ of $10 \% \mathrm{w} / \mathrm{v}$ methanolic $\mathrm{KOH}$. The tubes were flushed with nitrogen gas and capped before incubating at $70^{\circ} \mathrm{C}$ for $90 \mathrm{~min}$. Samples were cooled to room temperature before $1 \mathrm{ml}$ of water and $2 \mathrm{ml}$ of $\mathrm{n}$-hexane were added and vortexed. The hexane phase was transferred to glass vials and the extraction process was repeated. Combined extracts were dried under nitrogen and derivatized by adding $50 \mu \mathrm{l} \mathrm{N}$,OBis(trimethylsilyl)trifluoroacetamide:Trimethylchlorosilane (BSTFA, $1 \%$ TMCS) and incubating at $60^{\circ} \mathrm{C}$ for $30 \mathrm{~min}$. Derivatized samples were dried under nitrogen or by vacuum centrifugation for $\sim 30 \mathrm{~min}$, and finally suspended in ethyl acetate for GC-MS analysis.

\section{GC-MS analysis of sterols}

Derivatized sterol extracts and standards were analyzed on an Agilent Technologies 5977 GC/MSD equipped with a Agilent J\&W DB1MS UI capillary column with $45 \mathrm{~m}$ in length, $0.25 \mathrm{~mm}$ inner diameter and $0.25 \mu \mathrm{m}$ phase thickness (phase- 100\% dimethylpolysiloxane). Sterols from $1 \mu$ injections were separated using an initial oven temperature of $40^{\circ} \mathrm{C}$ for $1 \mathrm{~min}$ followed by a $20^{\circ} \mathrm{C} / \mathrm{min}$ ramp to $320^{\circ} \mathrm{C}$, which was held for $12 \mathrm{~min}$ (constant helium flow of $1 \mathrm{ml} / \mathrm{min}$ ). The mass spectrometer source and transfer line temperatures were set at $260^{\circ} \mathrm{C}$ and $280^{\circ} \mathrm{C}$, respectively and the GC inlet was operated in splitless mode. Mass spectral data was analyzed using MassHunter Workstation Software (Agilent). Parent and fragment ion counts were extracted at $129.3,454.3,456.3,458.3$, and $468.3 \mathrm{~m} / \mathrm{z}$ using a window of $+/-0.5 \mathrm{~m} / \mathrm{z}$ for analysis. Extracted Ion Chromatograms (EICs) were aligned, then 
individual sterols quantified as baseline-corrected peak areas across appropriate retention time windows for the following ions: 454.3, 7dehydrodesmosterol; 456.3, 7-dehydrocholesterol, zymosterol, 7dehydrolathosterol; 458.3, cholesterol, zymostenol+lathosterol; 468.3, ergosterol. Relative sterol abundances were calculated as the percentage of total ions detected for the set of measured sterols. Ambiguities between 7-dehydrocholesterol and desmosterol were resolved by examination of the 129/456 fragment ion ratio, and assignments confirmed using purified standards as shown in Supplementary Figure 4.

\section{Plate reader signaling assay}

Yeast were grown overnight in synthetic selective media and back-diluted 1:10 into media, with agonists as indicated, in Falcon 96 well microtiter plates to $100 \mu \mathrm{L}$ final volumes. Cells were shaken at $30^{\circ} \mathrm{C}$ for either 3 hours (alpha mating factor tests) or 8 hours (DAMGO tests) prior to measurement on a CLARIOstar plate reader (BMG Labtech). Values for OD600 and green fluorescence (excitation $469 \mathrm{~nm} \pm 13 \mathrm{~nm}$, emission $508 \mathrm{~nm} \pm 15 \mathrm{~nm}$ ) or red fluorescence (excitation $527 \mathrm{~nm} \pm 27$ $\mathrm{nm}$, emission $622 \mathrm{~nm} \pm 30 \mathrm{~nm}$ ) were collected for each sample.

\section{Flow cytometer signaling assay}

Overnight cultures grown in synthetic selective media were back-diluted 1:10 into fresh media containing the agonist being tested to a final volume of $100 \mu \mathrm{L}$ in a Falcon 96 well microtiter plate. Cells were shaken at $300 \mathrm{rpm}$ for 8 hours (or 6 hours for alpha mating factor tests) prior to measurement on an BD Accuri C6 flow cytometer. Either 10000 events, or those within $15 \mu \mathrm{L}$ of the culture, were recorded. For alpha mating factor response measurements the mean green fluorescence of the complete, ungated population was determined and used to calculate fold induction of fluorescence. Otherwise, within an experiment the biosensor that was brightest in its inactive state (no agonist) was used to establish an arbitrary green fluorescence intensity threshold such that $0.1-1 \%$ of cells were brighter than the threshold. This threshold was propagated to all conditions within the experiment and the percentage of the cells within each measurement that exceeded the threshold were recorded as the percentage of cells signaling. The percentage of cells signaling was exported to construct 4 parameter dose-response curves within Prism 8 (GraphPad) and calculate EC50s, IC50s and the maximum percentage of cells signaling within a biosensor-agonist condition.

\section{REFERENCES}

1. Pierce, K. L., Premont, R. T. \& Lefkowitz, R. J. Seven-transmembrane receptors. Nat. Rev. Mol. Cell Biol. 3, 639-650 (2002).

2. Hauser, A. S., Attwood, M. M., Rask-Andersen, M., Schioth, H. B. \& Gloriam, D. E. Trends in GPCR drug discovery: new agents, targets and indications. Nat. Rev. Drug Discov. 16, 829-842 (2017).

3. Elion, E. A. Pheromone response, mating and cell biology. Curr. Opin. Microbiol. 3, 573-581 (2000).

4. Alvaro, C. G. \& Thorner, J. Heterotrimeric G Protein-coupled Receptor Signaling in Yeast Mating Pheromone Response. J. Biol. Chem. 291, 7788-7795 (2016).

5. Brown, A. J. et al. Functional coupling of mammalian receptors to the yeast mating pathway using novel yeast/mammalian $G$ protein a-subunit chimeras. Yeast 16, 11-22 (2000).

6. King, K., Dohlman, H., Thorner, J., Caron, M. \& Lefkowitz, R. Control of yeast
Alternatively, for Figure 6, overnight cultures were backdiluted to an OD600 of approximately 0.2. The agonist was added upon dilution and cells were grown for 8 hours in 96-well deep well plates at a volume of $500 \mu \mathrm{l}$ at $30^{\circ} \mathrm{C}$ with shaking at $1000 \mathrm{rpm}$. 10000 cells of each sample were analyzed using a Sony SP6800 Spectral Analyzer.

\section{Microscopy}

Log phase yeast grown in synthetic selective media were mounted on slides and imaged using a DMi6000B microscope (Leica Microsystems) with an HCX PL APO 63x oil objective, an Orca R2 CCD camera (Hamamatsu) and Volocity software (PerkinElmer). Images were processed using FiJi ${ }^{90}$ and Photoshop CC (Adobe).

\section{Acknowledgements}

The authors thank Ian Riddington and the mass spectrometry facility at the University of Texas at Austin for their feedback and assistance with GC-MS sample preparation and analysis, and Josh Lutgens at the University of Texas at Austin for setting up and automating combinatorial DNA assembly using an Echo acoustic liquid handling robot. This study was financially supported by FRQNT Team and NSERC Discovery grants to V.J.J.M. and M.W.. B.D.M.B. was supported by a Concordia University Horizon Postdoctoral Fellowship, V.J.J.M. is supported by a Concordia University Senior Research Chair and M.W. is supported by a Canada Research Chair. Financial support was also provided by a Cooperative Agreement between the University of Texas at Austin and DEVCOM Army Research Laboratory to A.D.E., E.M.M., and J.D.G. (W911NF-17-2-0091). R.K.G was supported by the American Heart Association Predoctoral fellowship (\#18PRE34060258). E.M.M. acknowledges additional support from the Welch Foundation (F-1515); Army Research Office (W911NF-12-1-0390); and NIH (R35 GM122480).

\section{Contributions}

B.D.M.B., C.J.M., J.D.G., M.W., and V.J.J.M. designed the research. B.D.M.B, C.J.M, R.K.G., D.R.B., O.R, W.C., B.M.F., E.C.G., and E.M.M. performed the experiments. V.J.J.M., M.W., A.D.E., E.M.M., and J.D.G. supervised the research. B.D.M.B. and C.J.M. wrote the manuscript with editing help from V.J.J.M., J.D.G., M.W., E.M.M. and R.K.G.

\section{Conflict of interest}

The authors declare no competing interests.

mating signal transduction by a mammalian beta 2-adrenergic receptor and Gs alpha subunit. Science 250, 121-123 (1990).

7. Lengger, B. \& Jensen, M. K. Engineering G protein-coupled receptor signalling in yeast for biotechnological and medical purposes. FEMS Yeast Res. 20, 1-13 (2020).

8. Sarramegna, V., Talmont, F., Demange, P. \& Milon, A. Heterologous expression of G-protein-coupled receptors: comparison of expression systems from the standpoint of large-scale production and purification. Cell. Mol. Life Sci. CMLS 60, 1529-1546 (2003).

9. Lagane, B. et al. Role of Sterols in Modulating the Human $\mu$-Opioid Receptor Function in Saccharomyces cerevisiae. J. Biol. Chem. 275, 33197-33200 (2000).

10. O'Malley, M. A. et al. Progress toward heterologous expression of active Gprotein-coupled receptors in Saccharomyces cerevisiae: Linking cellular stress response with translocation and trafficking. Protein Sci. 18, 2356-2370 (2009).

11. Butz, J. A., Niebauer, R. T. \& Robinson, A. S. Co-expression of molecular chaperones does not improve the heterologous expression of mammalian Gprotein coupled receptor expression in yeast. Biotechnol. Bioeng. 84, 292-304 
(2003).

12. Yoo, J. I. \& O’Malley, M. A. Tuning Vector Stability and Integration Frequency Elevates Functional GPCR Production and Homogeneity in Saccharomyces cerevisiae. ACS Synth. Biol. 7, 1763-1772 (2018).

13. Schütz, M. et al. Directed evolution of $G$ protein-coupled receptors in yeast for higher functional production in eukaryotic expression hosts. Sci. Rep. 6, 21508 (2016).

14. Ritter, S. L. \& Hall, R. A. Fine-tuning of GPCR activity by receptor-interacting proteins. Nat. Rev. Mol. Cell Biol. 10, 819-830 (2009).

15. Chan, H. C. S. et al. Enhancing the Signaling of GPCRs via Orthosteric Ions. ACS Cent. Sci. 6, 274-282 (2020).

16. Opekarová, M. \& Tanner, W. Specific lipid requirements of membrane proteins-a putative bottleneck in heterologous expression. Biochim. Biophys. Acta BBA - Biomembr. 1610, 11-22 (2003).

17. Paila, Y. D. \& Chattopadhyay, A. Membrane Cholesterol in the Function and Organization of G-Protein Coupled Receptors. in Cholesterol Binding and Cholesterol Transport Proteins: (ed. Harris, J. R.) vol. 51 439-466 (Springer Netherlands, 2010).

18. Genheden, S., Essex, J. W. \& Lee, A. G. G protein coupled receptor interactions with cholesterol deep in the membrane. Biochim. Biophys. Acta BBABiomembr. 1859, 268-281 (2017).

19. Guixà-González, R. et al. Membrane cholesterol access into a G-proteincoupled receptor. Nat. Commun. 8, 14505 (2017).

20. Taghon, G. J., Rowe, J. B., Kapolka, N. J. \& Isom, D. G. Predictable cholesterol binding sites in GPCRs lack consensus motifs. Structure S0969212621000046 (2021) doi:10.1016/j.str.2021.01.004.

21. Morioka, S. et al. Effect of sterol composition on the activity of the yeast Gprotein-coupled receptor Ste2. Appl. Microbiol. Biotechnol. 97, 4013-4020 (2013).

22. Souza, C. M. et al. A stable yeast strain efficiently producing cholesterol instead of ergosterol is functional for tryptophan uptake, but not weak organic acid resistance. Metab. Eng. 13, 555-569 (2011).

23. Waldhoer, M., Bartlett, S. E. \& Whistler, J. L. Opioid Receptors. Annu. Rev. Biochem. 73, 953-990 (2004).

24. Skolnick, P. The Opioid Epidemic: Crisis and Solutions. Annu. Rev. Pharmacol. Toxicol. 58, 143-159 (2018).

25. Olesnicky, N. S., Brown, A. J., Dowell, S. J. \& Casselton, L. A. A constitutively active G-protein-coupled receptor causes mating self-compatibility in the mushroom Coprinus. EMBO J. 18, 2756-2763 (1999).

26. Erdman, S., Lin, L., Malczynski, M. \& Snyder, M. Pheromone-regulated Genes Required for Yeast Mating Differentiation. J. Cell Biol. 140, 461-483 (1998).

27. Heiman, M. G. \& Walter, P. Prm1p, a Pheromone-Regulated Multispanning Membrane Protein, Facilitates Plasma Membrane Fusion during Yeast Mating. J. Cell Biol. 151, 719-730 (2000).

28. Erickson, J. R. et al. Edg-2/Vzg-1 Couples to the Yeast Pheromone Response Pathway Selectively in Response to Lysophosphatidic Acid. J. Biol. Chem. 273, 1506-1510 (1998).

29. Casey, J. R., Grinstein, S. \& Orlowski, J. Sensors and regulators of intracellular pH. Nat. Rev. Mol. Cell Biol. 11, 50-61 (2010).

30. Harris, I., Jones, E. W. \& Aldred, C. N. The pH and Lactic Acid Content of the Cerebrospinal Fluid. J. Neurol. Neurosurg. Psychiatry s1-15, 46-52 (1934).

31. Edgar, R. C. MUSCLE: multiple sequence alignment with high accuracy and high throughput. Nucleic Acids Res. 32, 1792-1797 (2004).

32. Stevens, C., W. The evolution of vertebrate opioid receptors. Front. Biosci. 14, 1247 (2004).

33. Raynor, K. et al. Pharmacological characterization of the cloned kappa-, delta-, and mu-opioid receptors. Mol. Pharmacol. 45, 330-334 (1994).

34. Toll, L. et al. Standard binding and functional assays related to medications development division testing for potential cocaine and opiate narcotic treatment medications. NIDA Res. Monogr. 178, 440-466 (1998).

35. Nickolls, S. A., Waterfield, A., Williams, R. E. \& Kinloch, R. A. Understanding the Effect of Different Assay Formats on Agonist Parameters: A Study Using the $\mu$-Opioid Receptor. J. Biomol. Screen. 16, 706-716 (2011).

36. Kreil, G. et al. Deltorphin, a novel amphibian skin peptide with high selectivity and affinity for delta opioid receptors. Eur. J. Pharmacol. 162, 123-128 (1989).

37. Bilsky, J.et al. SNC 80, A Selective, Nonpeptidic and Systemically Active Opioid Delta Agonist. J. Pharmacol. Exp. Ther. 273, 359-366 (1995).
38. Camilleri, M. Novel pharmacology: asimadoline, a k-opioid agonist, and visceral sensation. Neurogastroenterol. Motil. 20, 971-979 (2008).

39. Yasuda, K. et al. Cloning and functional comparison of kappa and delta opioid receptors from mouse brain. Proc. Natl. Acad. Sci. 90, 6736 (1993).

40. Burford, N. T. et al. Identification of Selective Agonists and Positive Allosteric Modulators for $\mu$ - and $\delta$-Opioid Receptors from a Single High-Throughput Screen. J. Biomol. Screen. 19, 1255-1265 (2014).

41. Tonini, M. et al. Endomorphin-1 and endomorphin-2 activate $\mu$-opioid receptors in myenteric neurons of the guinea-pig small intestine: Naunyn. Schmiedebergs Arch. Pharmacol. 358, 686-689 (1998).

42. LaVigne, J., Keresztes, A., Chiem, D. \& Streicher, J. M. The endomorphin-1/2 and dynorphin-B peptides display biased agonism at the mu opioid receptor. Pharmacol. Rep. 72, 465-471 (2020).

43. Broad, J. et al. Human native kappa opioid receptor functions not predicted by recombinant receptors: Implications for drug design. Sci. Rep. 6, 30797 (2016).

44. Malatynska, E. et al. Human delta opioid receptor: functional studies on stably transfected Chinese hamster ovary cells after acute and chronic treatment with the selective nonpeptidic agonist SNC-80. J. Pharmacol. Exp. Ther. 278, 1083 (1996).

45. Knapp, R. J. et al. Structure-activity relationships for $\mathrm{SNC} 80$ and related compounds at cloned human delta and mu opioid receptors. J. Pharmacol. Exp. Ther. 277, 1284 (1996).

46. Quitterer, U., Pohl, A., Langer, A., Koller, S. \& AbdAlla, S. A cleavable signal peptide enhances cell surface delivery and heterodimerization of Ceruleantagged angiotensin II AT1 and bradykinin B2 receptor. Biochem. Biophys. Res. Commun. 409, 544-549 (2011).

47. Martoglio, B. \& Dobberstein, B. Signal sequences: more than just greasy peptides. Trends Cell Biol. 8, 410-415 (1998).

48. Rutz, C., Klein, W. \& Schülein, R. N-Terminal Signal Peptides of G ProteinCoupled Receptors. in Progress in Molecular Biology and Translational Science vol. 132 267-287 (Elsevier, 2015).

49. Fitzgerald, I. \& Glick, B. S. Secretion of a foreign protein from budding yeasts is enhanced by cotranslational translocation and by suppression of vacuolar targeting. Microb. Cell Factories 13, 1-12 (2014).

50. Mayer, P. \& Höllt, V. Allelic and somatic variations in the endogenous opioid system of humans. Pharmacol. Ther. 91, 167-177 (2001).

51. Olsen, T., Rasmussen, A., Kringen, MK \& Molden, E. A girl of early school-age with no response to opioids during general anaesthesia. Tidsskr. Den Nor. Legeforening 139, 6 .

52. Ravindranathan, A. et al. Functional characterization of human variants of the mu-opioid receptor gene. Proc. Natl. Acad. Sci. 106, 10811-10816 (2009).

53. Lötsch, J. \& Geisslinger, G. Are $\mu$-opioid receptor polymorphisms important for clinical opioid therapy? Trends Mol. Med. 11, 82-89 (2005).

54. Lemos Duarte, M. \& Devi, L. A. Post-translational Modifications of Opioid Receptors. Trends Neurosci. 43, 417-432 (2020).

55. Shiwarski, D. J., Crilly, S. E., Dates, A. \& Puthenveedu, M. A. Dual RXR motifs regulate nerve growth factor-mediated intracellular retention of the delta opioid receptor. Mol. Biol. Cell 30, 680-690 (2019).

56. Geva, Y. \& Schuldiner, M. The Back and Forth of Cargo Exit from the Endoplasmic Reticulum. Curr. Biol. 24, R130-R136 (2014).

57. Li, J.-G., Chen, C. \& Liu-Chen, L.-Y.N-Glycosylation of the Human $\kappa$ Opioid Receptor Enhances Its Stability but Slows Its Trafficking along the Biosynthesis Pathway. Biochemistry 46, 10960-10970 (2007).

58. Lackman, J. J., Markkanen, P. M. H., Hogue, M., Bouvier, M. \& Petäjä-Repo, U. E. N-Glycan-dependent and -independent Quality Control of Human $\delta$ Opioid Receptor N-terminal Variants. J. Biol. Chem. 289, 17830-17842 (2014).

59. Markkanen, P. M. H. \& Petäjä-Repo, U. E. N-Glycan-mediated Quality Control in the Endoplasmic Reticulum Is Required for the Expression of Correctly Folded $\delta$-Opioid Receptors at the Cell Surface. J. Biol. Chem. 283, 29086-29098 (2008).

60. Chaturvedi, K., Shahrestanifar, M. \& Howells, R. D. mu Opioid receptor: role for the amino terminus as a determinant of ligand binding affinity. Mol. Brain Res. 76, 64-72 (2000).

61. Deng, H. B. et al. Role for the C-Terminus in Agonist-Induced $\mu$ Opioid Receptor Phosphorylation and Desensitization. Biochemistry 39, 5492-5499 (2000).

62. Manglik, A. et al. Crystal structure of the $\mu$-opioid receptor bound to a 
bioRxiv preprint doi: https://doi.org/10.1101/2021.05.12.443385; this version posted May 14, 2021. The copyright holder for this preprint (which was not certified by peer review) is the author/funder. All rights reserved. No reuse allowed without permission.

morphinan antagonist. Nature 485, 321-326 (2012).

63. Feng, G.-J. et al. Selective Interactions between Helix VIII of the Human $\mu-$ Opioid Receptors and the C Terminus of Periplakin Disrupt G Protein Activation. J. Biol. Chem. 278, 33400-33407 (2003).

64. Platt, F. M. et al. Disorders of cholesterol metabolism and their unanticipated convergent mechanisms of disease. Annu Rev Genomics Hum Genet 15, 173194 (2014).

65. Ehrenworth, A. M., Claiborne, T. \& Peralta-Yahya, P. Medium-Throughput Screen of Microbially Produced Serotonin via a G-Protein-Coupled ReceptorBased Sensor. Biochemistry 56, 5471-5475 (2017).

66. Weston, C., Poyner, D., Patel, V., Dowell, S. \& Ladds, G. Investigating G protein signalling bias at the glucagon-like peptide-1 receptor in yeast. Br. J. Pharmacol. 171, 3651-3665 (2014).

67. Ishii, J. et al. Cell Wall Trapping of Autocrine Peptides for Human G- ProteinCoupled Receptors on the Yeast Cell Surface. PLoS ONE 7, 10 (2012).

68. Klein, C. et al. Identification of surrogate agonists for the human FPRL-1 receptor by autocrine selection in yeast. Nat. Biotechnol. 16, 1334-1337 (1998).

69. Jafurulla, M., Aditya Kumar, G., Rao, B. D. \& Chattopadhyay, A. A Critical Analysis of Molecular Mechanisms Underlying Membrane Cholesterol Sensitivity of GPCRs. Adv. Exp. Med. Biol. 1115, 21-52 (2019).

70. Gaibelet, G. et al. Cholesterol content drives distinct pharmacological behaviours of $\mu$-opioid receptor in different microdomains of the CHO plasma membrane. Mol. Membr. Biol. 25, 423-435 (2008).

71. Levitt, E. S., Clark, M. J., Jenkins, P. M., Martens, J. R. \& Traynor, J. R. Differential effect of membrane cholesterol removal on mu- and delta-opioid receptors: a parallel comparison of acute and chronic signaling to adenylyl cyclase. J. Biol. Chem. 284, 22108-22122 (2009).

72. Zheng, H. et al. Palmitoylation and membrane cholesterol stabilize $\mu$-opioid receptor homodimerization and $\mathrm{G}$ protein coupling. BMC Cell Biol. 13, 6 (2012).

73. Zhuang, Y. et al. Structure of formylpeptide receptor 2-Gi complex reveals insights into ligand recognition and signaling. Nat. Commun. 11, 885 (2020).

74. Michal, P., Rudajev, V., El-Fakahany, E. E. \& Doležal, V. Membrane cholesterol content influences binding properties of muscarinic M2 receptors and differentially impacts activation of second messenger pathways. Eur. J. Pharmacol. 606, 50-60 (2009).

75. Bari, M., Paradisi, A., Pasquariello, N. \& Maccarrone, M. Cholesteroldependent modulation of type 1 cannabinoid receptors in nerve cells. J. Neurosci. Res. 81, 275-283 (2005).

76. Albert, A. \& Boeszebattaglia, $\mathrm{K}$. The role of cholesterol in rod outer segment membranes. Prog. Lipid Res. 44, 99-124 (2005).

77. Paila, Y. D., Murty, M. R. V. S., Vairamani, M. \& Chattopadhyay, A. Signaling by the human serotonin1A receptor is impaired in cellular model of SmithLemli-Opitz Syndrome. Biochim. Biophys. Acta BBA - Biomembr. 1778, 15081516 (2008).

78. Rodriguez, R. E. et al. Characterization of ZFOR1, a putative delta-opioid receptor from the teleost zebrafish (Danio rerio). Neurosci. Lett. 4 (2000).

79. Sharpe, H. J., Stevens, T. J. \& Munro, S. A Comprehensive Comparison of Transmembrane Domains Reveals Organelle-Specific Properties. Cell 142, 158169 (2010).

80. Manglik, A. et al. Structure-based discovery of opioid analgesics with reduced side effects. Nature 537, 185-190 (2016).

81. Ehrlich, A. T. et al. Biased Signaling of the Mu Opioid Receptor Revealed in Native Neurons. iScience 14, 47-57 (2019).

82. Kroeze, W. K. et al. PRESTO-Tango as an open-source resource for interrogation of the druggable human GPCRome. Nat. Struct. Mol. Biol. 22, 362-369 (2015).

83. Pyne, M. E. et al. A yeast platform for high-level synthesis of tetrahydroisoquinoline alkaloids. Nat. Commun. 11, 3337 (2020).

84. Ostrov, N. et al. A modular yeast biosensor for low-cost point-of-care pathogen detection. Sci. Adv. 3, e1603221 (2017).

85. Brachmann, C. B. et al. Designer deletion strains derived from Saccharomyces cerevisiae S288C: A useful set of strains and plasmids for PCR-mediated gene disruption and other applications. Yeast 14, 115-132 (1998).

86. Lee, M. E., DeLoache, W. C., Cervantes, B. \& Dueber, J. E. A Highly Characterized Yeast Toolkit for Modular, Multipart Assembly. ACS Synth. Biol. 4, 975-986 (2015).
87. Ryan, O. W. et al. Selection of chromosomal DNA libraries using a multiplex CRISPR system. eLife 3, e03703 (2014).

88. Gietz, R. D. \& Schiestl, R. H. High-efficiency yeast transformation using the LiAc/SS carrier DNA/PEG method. Nat. Protoc. 2, 31-34 (2007).

89. Engler, C., Kandzia, R. \& Marillonnet, S. A One Pot, One Step, Precision Cloning Method with High Throughput Capability. PLOS ONE 3, e3647 (2008).

90. Schindelin, J.et al. Fiji: an open-source platform for biological-image analysis. Nat. Methods 9, 676-682 (2012). 